TRANSACTIONS OF THE

AMERICAN MATHEMATICAL SOCIETY

Volume 357, Number 3, Pages 1095-1124

S 0002-9947(04)03556-1

Article electronically published on July 16, 2004

\title{
GRÖBNER BASES OF ASSOCIATIVE ALGEBRAS AND THE HOCHSCHILD COHOMOLOGY
}

\author{
YUJI KOBAYASHI
}

\begin{abstract}
We give an algorithmic way to construct a free bimodule resolution of an algebra admitting a Gröbner base. It enables us to compute the Hochschild (co)homology of the algebra. Let $A$ be a finitely generated algebra over a commutative ring $K$ with a (possibly infinite) Gröbner base $G$ on a free algebra $F$, that is, $A$ is the quotient $F / I(G)$ with the ideal $I(G)$ of $F$ generated by $G$. Given a Gröbner base $H$ for an $A$-subbimodule $L$ of the free $A$-bimodule $A \cdot X \cdot A=A_{K} \otimes K \cdot X \otimes_{K} A$ generated by a set $X$, we have a morphism $\partial$ of $A$-bimodules from the free $A$-bimodule $A \cdot H \cdot A$ generated by $H$ to $A \cdot X \cdot A$ sending the generator $[h]$ to the element $h \in H$. We construct a Gröbner base $C$ on $F \cdot H \cdot F$ for the $A$-subbimodule $\operatorname{Ker}(\partial)$ of $A \cdot H \cdot A$, and with this $C$ we have the free $A$-bimodule $A \cdot C \cdot A$ generated by $C$ and an exact sequence $A \cdot C \cdot A \rightarrow A \cdot H \cdot A \rightarrow A \cdot X \cdot A$. Applying this construction inductively to the $A$-bimodule $A$ itself, we have a free $A$-bimodule resolution of $A$.
\end{abstract}

\section{INTRODUCTION}

The Gröbner base theory for polynomial rings has been developed since Buchberger [5], and has found vast fields of application. It is a central technique used in the computational theory of polynomial rings and algebraic geometry ([2], [5], [8]). Many authors have tried to generalize the theory to more general rings. For example, Mora [21] developed a Gröbner base theory for general associative algebras (the idea already appeared in Bergman [3]). But a shortcoming of the theory on non-commutative ring is that a finitely generated ideal may not have a finite Gröbner base, and so the Buchberger algorithm does not converge in general (see [9]). Some authors (for example Kandre-Rody and Weispfenning [13, see also [18]) developed the theory for certain restricted types of rings for which the Buchberger algorithm works. But even if an ideal has no finite Gröbner base, if it has an infinite (but well-behaved in some sense) one, it is sometimes useful.

In the present paper we consider a possibly infinite Gröbner base $G$ on a free algebra $F$ over a commutative ring $K$. We give an algorithmic way to construct a free bimodule resolution over the quotient algebra $A=F / I(G)$, where $I(G)$ is the ideal of $F$ generated by $G$. It enables us to compute the Hochschild cohomology (Hochschild [1]) of $A$. In the construction Gröbner bases on free $F$-bimodules play a crucial role. We discuss Gröbner bases on free algebras and on free bimodules from a stand point of rewriting systems. Actually, the theory of Gröbner bases and

Received by the editors September 10, 2002 and, in revised form, September 9, 2003.

2000 Mathematics Subject Classification. Primary 16E05, 16E40, 16 S15. 
that of rewriting systems are considered to be equivalent (in certain situations, see for example Madlener and Reinert [20]).

Let $G$ be a set of monic elements of a free algebra $F$ over $K$, here $f \in F$ is monic if the coefficient of the leading term $\operatorname{lt}(f)$ of $f$ with respect to some fixed term order is 1. $G$ is a Gröbner base of the ideal $I(G)$ if the associated system $R_{G}=\{\operatorname{lt}(g) \rightarrow-\operatorname{rt}(g) \mid g \in G\}$ is a complete (noetherian and confluent) rewriting system, where $\operatorname{rt}(g)=g-\operatorname{lt}(g)$. If $K$ is a field, every ideal of $F$ admits a (possibly infinite) Gröbner base.

Let $A$ be an algebra over $K$ admitting a Gröbner base $G$ on $F$, that is, $A$ is the quotient $F / I(G)$. Moreover, we consider a Gröbner base $H$ for an $A$-subbimodule $L$ of the free $A$-bimodule $A \cdot X \cdot A=A_{K} \otimes K \cdot X \otimes_{K} A$ generated by a set $X$. $H$ is a generating set for $L$ of monic elements in the free $F$-bimodule $F \cdot X \cdot F$ generated by $X$ such that the system $T_{H}=\{\operatorname{lt}(h) \rightarrow-\operatorname{rt}(h) \mid h \in H\}$ is a complete rewriting system on $F \cdot X \cdot F$ modulo $G$ (see Section 3 for a precise definition). We have a morphism $\partial$ of $A$-bimodules from the free $A$-bimodule $A \cdot H \cdot A$ generated by $H$ to $A \cdot X \cdot A$ given by $\partial([h])=h$, where $[h]$ is the formal generator corresponding to $h \in H$.

The key technique in this paper lies in a construction of a Gröbner base $C$ on $F \cdot H \cdot F$ for the $A$-subbimodule $\operatorname{Ker}(\partial)$ of $A \cdot H \cdot A$ (Section 5 ). This $C$ is made from the critical pairs of reductions on $F \cdot X \cdot F$ with respect to $H$ and $G$, and thus, if $G$ and $H$ are effectively given, $C$ can be effectively constructed. With this $C$ we have the free $A$-bimodule $A \cdot C \cdot A$ generated by $C$ and an exact sequence $A \cdot C \cdot A \rightarrow A \cdot H \cdot A \rightarrow A \cdot X \cdot A$ (Section 6 ). Applying this construction inductively to the $A$-bimodule $A$ itself, we have a free $A$-bimodule resolution of $A$ (Section 7). This is considered to be a two-sided version of the construction given by Anick [1] (see also Kobayashi [16]), and, when $\mathrm{A}$ is supplemented, taking the functor $\otimes_{A} K$ on the right-hand side of our resolution yields a left free resolution of the left $A$ module $K$. The last section is devoted to exhibit some examples which show the effectiveness of our methods.

The technique developed in this paper on free algebras and free bimodules can be generalized to path algebras and projective bimodules. This will be discussed in a forthcoming paper.

\section{GRÖBNER BASES FOR ALGEBRAS}

Let $\Sigma$ be a (finite) alphabet and let $\Sigma^{*}$ be the free monoid generated by $\Sigma$, that is, $\Sigma^{*}$ is the set of words over $\Sigma$ and the monoid operation of it is given by the concatenation of words. The empty word, denoted by 1 in this paper, is the identity element of $\Sigma^{*}$. The set of words of length $n$ and the set of words of length $\leq n$ are denoted by $\Sigma^{n}$ and $\Sigma^{\leq n}$, respectively, that is, $\Sigma^{n}=\left\{x \in \Sigma^{*}|| x \mid=n\right\}$ and $\Sigma^{\leq n}=\left\{x \in \Sigma^{*}|| x \mid \leq n\right\}$, where $|x|$ denotes the length of a word $x$. Accordingly, $\Sigma^{0}=\{1\}, \Sigma^{1}=\Sigma, \Sigma^{\leq n}=\bigcup_{0 \leq i \leq n} \Sigma^{i}$, and $\Sigma^{*}=\bigcup_{0 \leq i} \Sigma^{i}$.

Let $K$ be a commutative ring with 1 and let $F=\bar{K} \cdot \Sigma^{*}$ be the free (associative) algebra generated by $\Sigma$ over $K . \quad F$ is the free $K$-module spanned by $\Sigma^{*}$ with the multiplication induced by the monoid operation of $\Sigma^{*}$. An element $f$ of $F$ is uniquely written as

$$
f=\sum_{i=1}^{n} k_{i} x_{i}
$$


with $k_{i} \in K \backslash\{0\}$ and different elements $x_{i}$ in $\Sigma^{*}$. We fix a compatible well-order $\succ$ on $\Sigma^{*}$, that is, $\succ$ is a (strict) total order on $\Sigma^{*}$ such that there is no infinite decreasing sequence $x_{1} \succ x_{2} \succ \ldots$ and for any $x, y, z \in \Sigma^{*}, x \succ y$ implies $x z \succ y z$ and $z x \succ z y$. A typical such order is the length-lexicographic order $\succ_{\text {llex }}$, namely, $x \succ_{\text {llex }} y$ if either $|x|>|y|$, or $|x|=|y|$ and $y$ is smaller than $x$ in the lexicographic order based on a pre-given linear order on $\Sigma$.

Let $f$ be an element of $F$ written as (1). If $x_{1}$ is the maximal among $x_{i}(i=$ $1, \ldots, n)$ with respect to $\succ, k_{1} x_{1}$ is called the leading term of $f$ and $\operatorname{denoted}$ by $\operatorname{lt}(f)$. Let $\operatorname{rt}(f)=f-\operatorname{lt}(f)$. We extend the order $\succ$ on $\Sigma^{*}$ to a (partial) order on $F$, which is also denoted by $\succ$, as follows. First, $f \succ 0$ for any $f \neq 0$. Let $f$ and $g$ be nonzero elements in $F$ and let $\operatorname{lt}(f)=k \cdot x$ and $\operatorname{lt}(g)=\ell \cdot y$ with $k, \ell \in K \backslash\{0\}$ and $x, y \in \Sigma^{*}$. Define $f \succ g$ if and only if either $x \succ y$ or $x=y$ and $\operatorname{rt}(f) \succ \operatorname{rt}(g)$. It is easy to see that $\succ$ is also well-founded on $F$, that is, there is no infinite sequence $f_{1} \succ f_{2} \succ \cdots$ in $F$.

A (monic) rewriting rule is a pair $(u, v)$, where $u \in \Sigma^{*}$ and $v \in F$ with $u \succ v$. A rule $(u, v)$ is written as $u \rightarrow v$. A (monic) rewriting system $R$ is a (not necessarily finite) set of rewriting rules. If $f \in F$ has a nonzero term $k \cdot x$ and $x=x_{1} u x_{2}$ with $x_{1}, x_{2} \in \Sigma^{*}$ and $u \rightarrow v \in R$, the rule $u \rightarrow v$ can be applied to $f$ and $f$ is transformed (or rewritten) to $g=f-k \cdot x_{1}(u-v) x_{2}$. In this situation we write $f \rightarrow_{R} g$, and we call $\rightarrow_{R}$ the one-step reduction by $R$.

Let $\rightarrow{ }_{R}^{*}$ denote the reflexive transitive closure of the one-step reduction relation $\rightarrow_{R}$, and let $\leftrightarrow_{R}^{*}$ be the reflexive symmetric and transitive closure of $\rightarrow_{R}$. Set $G_{R}=\{u-v \mid u \rightarrow v \in R\}$, and let $I(R)$ be the (two-sided) ideal of $F$ generated by $G_{R}$.

Here we depict the following basic results.

Proposition 2.1. The relation $\leftrightarrow_{R}^{*}$ is equal to the congruence on $F$ modulo $I(R)$, that is,

$$
f \leftrightarrow{ }_{R}^{*} g \Leftrightarrow f \equiv g(\bmod I(R))
$$

for $f, g \in F$. In particular,

$$
f \leftrightarrow{ }_{R}^{*} 0 \Leftrightarrow f \equiv 0(\bmod I(R))
$$

for $f \in F$, that is,

$$
I(R)=\left\{f \in F \mid f \leftrightarrow_{R}^{*} 0\right\} .
$$

The quotient algebra $A=F / I(R)=F / \leftrightarrow_{R}^{*}$ is said to be defined by the rewriting system $R$. Two rewriting systems $R_{1}$ and $R_{2}$ on $F$ are equivalent if $\leftrightarrow_{R_{1}}^{*}=\leftrightarrow_{R_{2}}^{*}$. The equivalent systems define the same quotient, and thus, by Proposition 2.1, $R_{1}$ and $R_{2}$ are equivalent if and only if $I\left(R_{1}\right)=I\left(R_{2}\right)$.

If $f \rightarrow_{R} g$, then, as easily seen, $f \succ g$ by the compatibility of $\succ$. Since $\succ$ is wellfounded, the relation $\rightarrow_{R}$ is noetherian (terminating), that is, there is no infinite sequence $f_{1} \rightarrow_{R} f_{2} \rightarrow_{R} \cdots \rightarrow_{R} f_{n} \rightarrow_{R} \cdots$ in $F$. If two elements $f$ and $g$ of $F$ have a common $R$-descendant, that is, there is $h \in F$ such that $f \rightarrow{ }_{R}^{*} h$ and $f \rightarrow{ }_{R}^{*} h$, we say that $f \downarrow_{R} g$ holds. $R$ is called confluent if for any $f, g, h \in F$ such that $h \rightarrow_{R}^{*} f$ and $h \rightarrow_{R}^{*} g, f \downarrow_{R} g$ holds. In general, a noetherian and confluent system is called complete, but in our situation here, a confluent system is complete because a rewriting system we consider is always noetherian. 
Lemma 2.2. For $f, g \in F, f-g \rightarrow_{R}^{*} 0$ implies $f \downarrow_{R} g$.

Proof. Let $h=f-g$ and suppose $h \rightarrow_{R}^{*} 0$. We shall show $f \downarrow_{R} g$ by induction on $h$ with respect to the well-order $\succ$. If $h=0$, then $f=g$ and $f \downarrow_{R} g$ trivially holds. If $h \neq 0$, then $h \rightarrow_{R} h^{\prime} \rightarrow_{R}^{*} 0$, where $h$ has a term $k \cdot x$ with $k \in K \backslash\{0\}$ and $x \in \Sigma^{*}$ such that $x \rightarrow_{R} x^{\prime}$ and $h^{\prime}=h-k \cdot\left(x-x^{\prime}\right)$. Because $h=f-g$, we have $k=k_{1}-k_{2}$, where $k_{1} \cdot x$ and $k_{2} \cdot x$ are terms of $f$ and $g$ respectively (one of $k_{1}$, $k_{2} \in K$ is possibly zero). Let $f^{\prime}=f-k_{1}\left(x-x^{\prime}\right)$ and $g^{\prime}=g-k_{2}\left(x-x^{\prime}\right)$, then, $f \rightarrow_{R} f^{\prime}$ (or $f=f^{\prime}$ if $k_{1}=0$ ), $g \rightarrow_{R} g^{\prime}$ (or $g=g^{\prime}$ if $k_{2}=0$ ) and $h^{\prime}=f^{\prime}-g^{\prime}$. Since $h \succ h^{\prime}$, we see $f^{\prime} \downarrow_{R} g^{\prime}$ by the induction hypothesis, and consequently we have $f \downarrow_{R} g$.

The converse implication in Lemma 2.2 is not true in general. For example, consider the rewriting system $R=\{a b \rightarrow a, b a \rightarrow b\}$ over the alphabet $\Sigma=\{a, b\}$ and let $f=a b a+a$ and $g=a b a+a a$. Then, $f \rightarrow_{R} a a+a$ and $g \rightarrow_{R} a b+a a \rightarrow_{R}$ $a+a a$, that is, $f \downarrow_{R} g$ holds. But $f-g=a-a a$ cannot be reduced to 0. However, if $R$ is confluent, the converse is also true (see Proposition 2.3 below).

An element $f$ in $F$ is irreducible (R-irreducible to specify $R$ ) if there is no $g$ such that $f \rightarrow_{R} g$. In particular, an irreducible monic monomial $x \in \Sigma^{*}$ is called an irreducible word, and $\operatorname{Irr}(R)$ denotes the set of irreducible words. Clearly, $\operatorname{Irr}(R)=$ $\Sigma^{*} \backslash \Sigma^{*} \cdot \operatorname{Dom}(R) \cdot \Sigma^{*}$, where $\operatorname{Dom}(R)=\{u \mid u \rightarrow v \in R\}$, and $f \in F$ is irreducible if and only if $f$ is a $K$-linear combination of irreducible words. An element $f \in F$ is $R$-reducible if it is not $R$-irreducible. A word $x$ is a minimal $R$-reducible word if it is $R$-reducible but any proper prefix of $x$ is $R$-irreducible.

Since $R$ is noetherian, for any $f \in F$ there is an irreducible $\hat{f} \in F$ such that $f \rightarrow_{R}^{*} \hat{f}$. If, moreover, $R$ is confluent (so complete), such an $\hat{f}$ is unique and is called the normal form of $f$, and, as is well known in the theory of rewriting systems, $f \leftrightarrow_{R}^{*} g$ if and only if $\hat{f}=\hat{g}$ (Proposition 2.3 below).

A subset $G$ of $F$ is monic if every $g \in G$ is monic, that is, the leading coefficient of $g$ is 1 . Let $I$ be an ideal of $F$. A set $G$ of generators of $I$ is called a Gröbner base of $I$, if it is monic and the system $R_{G}=\{\operatorname{lt}(g) \rightarrow-\operatorname{rt}(g) \mid g \in G\}$ associated with $G$ is a complete rewriting system. We usually confuse a Gröbner base $G$ with the associated rewriting system $R_{G}$. Accordingly, when we write $g=u-v \in G$, we implicitly assume that $u=\operatorname{lt}(g)$ and $v=-\operatorname{rt}(g)$, and we just write $\rightarrow_{G}$ for the relation $\rightarrow R_{G}$, for example. We say $f \in F$ is $G$-irreducible if it is $R_{G}$-irreducible, and $\operatorname{Dom}(G)$ and $\operatorname{Irr}(G)$ denote $\operatorname{Dom}\left(R_{G}\right)$ and $\operatorname{Irr}\left(R_{G}\right)$ respectively.

We depict the fundamental results on complete rewriting systems and Gröbner bases as follows (see [2], 4], [6], [8]).

Proposition 2.3. Let $G$ be a Gröbner base of an ideal $I$ of $F$, let $A=F / I$ be the quotient algebra of $F$ by $I$, and let $\rho: F \rightarrow A$ be the canonical surjection. Then, $\rho$ is injective on $\operatorname{Irr}(G)$ and $\rho(\operatorname{Irr}(G))$ forms a linear $K$-base of $A=F / I$. Any $f$ has the unique normal form $\hat{f}$, and we have

$$
\hat{f}=\hat{g} \Leftrightarrow f \leftrightarrow_{G}^{*} g \Leftrightarrow f-g \rightarrow_{G}^{*} 0 \Leftrightarrow \rho(f)=\rho(g)
$$

for any $f, g \in F$. In particular, we have

$$
I=\{f \in F \mid \hat{f}=0\}=\left\{f \in F \mid f \rightarrow_{G}^{*} 0\right\} .
$$


Corollary 2.4. If $G$ is a Gröbner base, the mapping ^ sending $f \in F$ to the normal form $\hat{f} \in F$ is $K$-linear;

$$
(k \cdot f+\ell \cdot g) \hat{)}=k \cdot \hat{f}+\ell \cdot \hat{g}
$$

for $k, \ell \in K$ and $f, g \in F$.

An algebra $A$ over $K$ is said to admit a Gröbner base if it is isomorphic to the quotient $F / I$ of some finitely generated free algebra $F$ over $K$ modulo an ideal $I$ with a Gröbner base. By Proposition 2.3 we have

Corollary 2.5. An algebra over $K$ admitting a Gröbner base is free as a $K$-module.

Example 2.6. (1) A finitely generated algebra $A$ over a field $K$ admits a Gröbner base. In fact, any ideal $I$ of a free algebra $F=K \Sigma^{*}$ over $K$ has a Gröbner base with respect to a fixed compatible well-order $\succ$ on $\Sigma^{*}$. If $x \in \Sigma^{*}$ is a linear combination of $x_{i} \in \Sigma^{*}$ with $x_{i} \prec x$ over $K$ modulo $I$;

$$
x=\sum k_{i} x_{i}(\bmod I), \quad k_{i} \in K \backslash\{0\},
$$

choose one such combination and consider the element $x-\sum k_{i} x_{i}$. Let $G$ be the set of all such elements. Then, $G$ is a Gröbner base of $I$.

(2) A monoid algebra $A=K \cdot M$ of a finitely generated monoid $M$ over a commutative ring $K$ admits a Gröbner base. Let $\Sigma$ be a set of generators and $\rho: \Sigma^{*} \rightarrow M$ the surjection. Let $I$ be the kernel of the natural morphism $K \Sigma^{*} \rightarrow A$ induced by $\rho$. For $x \in \Sigma^{*}, \hat{x}$ denotes the minimal element in $\Sigma^{*}$ with respect to a fixed well-order $\succ$ on $\Sigma^{*}$ in the congruence class $\rho^{-1}(\rho(x))$ of $x$. Then, $G=\left\{x-\hat{x} \mid x \in \Sigma^{*}, x \neq \hat{x}\right\}$ forms a Gröbner base of $I$.

(3) The enveloping algebra $A$ of a Lie algebra $L$ over $K$ ( $L$ is free as a $K$-module) admits a Gröbner base. Let $\Sigma$ be a linear base of $L$ over $K$. Then, $A=K \Sigma^{*} / I$ for an ideal $I$ of $K \Sigma^{*}$. Let $>$ be a linear order on $\Sigma$. Then, $G=\{a b-b a-[a, b] \mid a, b \in$ $\Sigma, a>b\}$ forms a Gröbner base of $I$.

Remark 2.7. In Example 2.6, the Gröbner bases $G$ in (1) and (2) are usually infinite. The Gröbner base in (3) is finite if $\Sigma$ is finite.

Let $R$ be a rewriting system on $F=K \Sigma^{*}$. Let $u_{1} \rightarrow v_{1}, u_{2} \rightarrow v_{2} \in R$. Suppose $u_{1}$ overlaps properly with $u_{2}$, that is, $u_{1}=u_{1}^{\prime} z, u_{2}=z u_{2}^{\prime}$ with $u_{1}^{\prime}, u_{2}^{\prime}, z(\neq 1) \in \Sigma^{*}$. We have two reductions $p_{1}: u_{1} u_{2}^{\prime} \rightarrow v_{1} u_{2}^{\prime}$ and $p_{2}: u_{1}^{\prime} u_{2} \rightarrow u_{1}^{\prime} v_{2}$ applying the rules to $u_{1} u_{2}^{\prime}=u_{1}^{\prime} u_{2}$ in two different ways. We call $\left(v_{1} u_{2}^{\prime}, u_{1}^{\prime} v_{2}\right)$ a critical pair of elements of overlapping type and $\left(p_{1}, p_{2}\right)$ a critical pair of reductions. Next suppose $u_{1}$ contains $u_{2}$ as a subword, that is, $u_{1}=u^{\prime} u_{2} u^{\prime \prime}$ with $u^{\prime}, u^{\prime \prime} \in \Sigma^{*}$. Applying the rules to $u_{1}$ in two ways, we have a critical pair $\left(v_{1}, u^{\prime} v_{2} u^{\prime \prime}\right)$ of elements of inclusion type and a critical pair $\left(u_{1} \rightarrow v_{1}, u^{\prime} u_{2} u^{\prime \prime} \rightarrow u^{\prime} v_{2} u^{\prime \prime}\right)$ of reductions. For a critical pair $\left(x_{1}, x_{2}\right)$ of elements the difference $x_{1}-x_{2}$ is called an $S$-polynomial. A critical pair $\left(x_{1}, x_{2}\right)$ is resolvable if $x_{1} \downarrow_{R} x_{2}$.

Since our system $R$ is noetherian, $R$ is complete, if it is locally confluent, that is, for any $f, g, h \in F, h \rightarrow f$ and $h \rightarrow g$ imply $f \downarrow g$ ([4, [12]). The fundamental result in the theory of rewriting systems is a so-called critical pair theorem, which is a base of the completion procedure. The theorem asserts that a system is locally confluent if all the critical pairs are resolvable. Because our situation is a little different from the ones discussed in the literature, we give a proof sketch of the result here. 
Proposition 2.8. A system $R$ is complete if all the critical pairs for $R$ are resolvable.

Proof. We shall show that for any $f, g, h \in F, h \rightarrow f$ and $h \rightarrow g$ imply $f \downarrow g$ by induction on $h$ with respect to the order $\succ$ under the assumption that all the critical pairs are resolvable. From the induction hypothesis that $h^{\prime} \rightarrow f^{\prime}, h^{\prime} \rightarrow g^{\prime}$ and $h^{\prime} \prec h$ imply $f^{\prime} \downarrow g^{\prime}$, we can easily show that

(i) $h^{\prime} \rightarrow^{*} f^{\prime}, h^{\prime} \rightarrow^{*} g^{\prime}$ imply $f^{\prime} \downarrow g^{\prime}$ for any $f^{\prime}, g^{\prime}, h^{\prime} \in F$ such that $h^{\prime} \prec h$.

The following assertions can be proved by easy and direct calculations.

(ii) If two reductions $h \rightarrow f$ and $h \rightarrow g$ are made through applications of rules of $R$ to different terms of $h \in F$, then $f \downarrow g$.

(iii) If $f \rightarrow^{*} g$ for $f, g \in F$, then $k x f y \rightarrow^{*} k x g y$ for any $x, y \in \Sigma^{*}$ and $k \in K$.

(iv) If $f \rightarrow^{*} g$ for $f, g \in F$, then $f+h \rightarrow^{*} g+h$ for any irreducible $h \in F$.

(v) If two reductions $x \rightarrow f$ and $x \rightarrow g$ are made through applications of rules of $R$ to disjoint subwords of $x \in \Sigma^{*}$, then $f \downarrow g$.

Now, suppose $h \rightarrow f$ and $h \rightarrow g$ for $f, g, h \in F$. By (ii), in order to show $f \downarrow g$, we may suppose that $h \rightarrow f$ and $h \rightarrow g$ are through applications of rules to the same term $k x\left(k \in K, x \in \Sigma^{*}\right)$ of $h$. If $h$ has a reducible term other than $k x$, then $h \rightarrow h^{\prime}$ applying a rule to that term. Again by (ii), there are $f_{1}, g_{1} \in F$ such that $f \rightarrow^{*} f_{1}, h^{\prime} \rightarrow^{*} f_{1}, g \rightarrow^{*} g_{1}$ and $h^{\prime} \rightarrow^{*} g_{1}$. Since $h^{\prime} \prec h$, we have $f_{1} \downarrow g_{1}$ by (i). Consequently, we see that $f \downarrow g$ holds.

Therefore, we may assume that $h=k x+h^{\dagger}$ with irreducible $h^{\dagger} \in F$. If rules are applied to disjoint subwords of $x$ and we obtain reductions $k x \rightarrow t$ and $k x \rightarrow t^{\prime}$, then $t \downarrow t^{\prime}$ by (iii) and (v). Because $f=t+h^{\dagger}$ and $g=t^{\prime}+h^{\dagger}$, we have $f \downarrow g$ by (iv). Otherwise, $f=k x^{\prime} c_{1} x^{\prime \prime}+h^{\dagger}$ and $g=k y^{\prime} c_{2} y^{\prime \prime}+h^{\dagger}$ for some critical pair $\left(c_{1}, c_{2}\right)$, where $x^{\prime}, x^{\prime \prime}, y^{\prime}, y^{\prime \prime} \in \Sigma^{*}$. Since $c_{1} \downarrow c_{2}$ by assumption, we have $f \downarrow g$ in the same way as above.

Proposition 2.9. For a rewriting system $R$ the following statements are equivalent.

(1) $R$ is complete.

(2) Every critical pair is resolvable.

(3) Every $S$-polynomial is reduced to 0.

(4) Every element in $I(R)$ is reduced to 0.

Proof. $(2) \Rightarrow(1)$ : Proposition 2.8 .

$(1) \Rightarrow(4)$ : Proposition 2.3.

$(4) \Rightarrow(3)$ : Because every $S$-polynomial is in $I(R)$, it is reduced to 0 .

$(3) \Rightarrow(2)$ : By Lemma 2.2, $x_{1}-x_{2} \rightarrow{ }_{R}^{*} 0$ implies $x_{1} \downarrow_{R} x_{2}$ for any critical pair $\left(x_{1}, x_{2}\right)$.

A rewriting system $R$ is normalized, if the right-hand side $v$ of any rule $u \rightarrow v$ of $R$ is $R$-irreducible and the left-hand side $u$ is $(R \backslash\{u \rightarrow v\})$-irreducible. A set $G$ of monic elements is normalized if $R_{G}$ is also. For any Gröbner base $G$ we can always get a normalized Gröbner base equivalent to $G$ as follows.

Proposition 2.10. For any Gröbner base $G$ on $F$, there exists a normalized Gröbner base $G^{\prime}$ equivalent to $G$. If $G$ is finite, we can choose $G^{\prime}$ to be finite.

Proof. First, if there are more than one elements in $G$ with the same leading term, take just one of them and throw out the others. Then, for each $u-v$ remaining in $G$, if $u$ is reducible with respect to $(G \backslash\{u-v\})$, remove it from $G$; on the other hand, if $u$ is $(G \backslash\{u-v\})$-irreducible but $v$ is $G$-reducible, then replace it by $u-\hat{v}$, 
where $\hat{v}$ is the normal form of $v$ with respect $G$. We shall show that the set $G^{\prime}$ reformed from $G$ in this way is a normalized Gröbner base equivalent to $G$.

Let $I$ and $I^{\prime}$ be the ideals generated by $G$ and $G^{\prime}$ respectively. It is easy to see that $G^{\prime} \subset I$ and hence $I^{\prime} \subset I$. We shall show that $f \rightarrow_{G^{\prime}}^{*} 0$ for every $f \in I$. Let $f \in I$ and let $f^{\prime}$ be the irreducible descendant of $f$ with respect to $G^{\prime} ; f \rightarrow_{G^{\prime}}^{*} f^{\prime}$. Since $f-f^{\prime} \in I^{\prime} \subset I$, we see $f^{\prime} \in I$. Since $R_{G}$ is complete, $f^{\prime} \rightarrow_{G}^{*} 0$ by Proposition 2.3. So, if $f^{\prime} \neq 0$, a rule from $R_{G}$ must be applied to the leading term $\operatorname{lt}\left(f^{\prime}\right)=k \cdot x$ $\left(k \in K \backslash\{0\}, x \in \Sigma^{*}\right)$ of $f^{\prime}$, that is, $x=x_{1} u x_{2}, u \rightarrow v \in R_{G}$. If $u \in \operatorname{Irr}(G \backslash\{u-v\})$, then $u-\hat{v} \in G^{\prime}$ contradicting $G^{\prime}$-irreducibility of $f^{\prime}$. If $u \notin \operatorname{Irr}(G \backslash\{u, v\})$, there is an element $u^{\prime}-v^{\prime} \in G$ different from $u-v$ such that $u^{\prime}$ is a factor of $u ; u=u_{1} u^{\prime} u_{2}$ with $u_{1}, u_{2} \in \Sigma^{*}$ and $u^{\prime}-\hat{v}^{\prime} \in G^{\prime}$. This again contradicts the $G^{\prime}$-irreducibility of $f^{\prime}$. Hence, we have $f^{\prime}=0$ and $f \rightarrow_{G^{\prime}}^{*} 0$.

What we have shown above implies that $I=I^{\prime}$ and $R_{G^{\prime}}$ is complete by Proposition 2.9. Clearly $G^{\prime}$ is normalized by construction and it is finite if $G$ is also.

Note that if $G$ is normalized, there is no critical pair of inclusion type. In fact, for any $u-v \in G$, there is no $u^{\prime}-v^{\prime} \in G$ different from $u-v$ such that $u^{\prime}$ is a subword of $u$, because $u$ is $G \backslash\{u-v\}$-irreducible.

\section{GRÖBNER BASES FOR BIMODULES}

Let $K$ be a commutative ring with 1 . Let $A$ be a $K$-algebra. An $A$-bimodule $M$ is a $K$-module with left $A$-action and right $A$-action satisfying

$$
(a \cdot x) \cdot b=a \cdot(x \cdot b),
$$

for $a, b \in A, x \in M$, and the restrictions of left and right actions to $K$ coincide with the original $K$-module action. Let $A^{\circ}$ be the opposite algebra of $A$. An $A$-bimodule $M$ is naturally a left module over the enveloping algebra $A \otimes_{K} A^{\circ}$ with the left action

$$
(a \otimes b) \cdot x=a \cdot x \cdot b
$$

for $a \otimes b \in A \otimes_{K} A^{\circ}$ and $x \in M$. Conversely, a left $A \otimes_{K} A^{\circ}$-module $M$ is considered to be an $A$-bimodule.

An $A$-bimodule is free, if it is free when we regard it as left $A \otimes_{K} A^{\circ}$-module. The free $A$-bimodule $M$ generated by a set $X$ is isomorphic to

$$
A \otimes_{K} K X \otimes_{K} A,
$$

where $K X$ is the free $K$-module generated by $X$. An element $f$ of $M$ is written as a finite sum

$$
f=\sum a_{i} \otimes \xi_{i} \otimes b_{i}
$$

with $a_{i}, b_{i} \in A$ and $\xi_{i} \in X$, and for $a, b \in A$ we have

$$
a \cdot f \cdot b=\sum a a_{i} \otimes \xi_{i} \otimes b_{i} b .
$$

The free bimodule $M$ is written simply as $A \cdot X \cdot A$ and the element $f$ in (2) is written as $\sum a_{i}\left[\xi_{i}\right] b_{i}$.

Now, let $A$ be an algebra over $K$ admitting a Gröbner base and let $\Sigma$ be an alphabet corresponding to the generating set of $A$. Let $F=K \Sigma^{*}$ be the free algebra generated by $\Sigma$ over $K$ and let $\rho: F \rightarrow A$ be the surjection. Let $G$ be a 
normalized Gröbner base of $I=\operatorname{Ker}(\rho)$ with respect to a fixed compatible wellorder $\succ$ on $\Sigma^{*}$. In the rest of this section we discuss rewriting systems on free $F$-bimodules in this situation.

Let $X$ be a nonempty but possibly infinite set and we consider the free $F$ bimodule $F \cdot X \cdot F$ generated by $X$. Actually, $F \cdot X \cdot F$ is the free $K$-module generated by $\Sigma^{*} \times X \times \Sigma^{*}$ with two-sided $F$-action. Let $\succ$ be a well-order on the set of terms $x[\xi] y \in \Sigma^{*} \times X \times \Sigma^{*}$ with $x, y \in \Sigma^{*}$ and $\xi \in X$, which is compatible, that is, $f \succ f^{\prime}$ in $\Sigma^{*} \times X \times \Sigma^{*}$ implies $a \cdot f \cdot b \succ a \cdot f^{\prime} \cdot b$ for any $a, b \in \Sigma^{*}, a \succ a^{\prime}$ in $\Sigma^{*}$ implies $a \cdot f \succ a^{\prime} \cdot f$ for any $f \in \Sigma^{*} \times X \times \Sigma^{*}$ and $b \succ b^{\prime}$ implies $f \cdot b \succ f \cdot b^{\prime}$ for any $f \in \Sigma^{*} \times X \times \Sigma^{*}$. For example, starting with a linear order $>$ on $X$, we can define an order $\succ$ on $\Sigma^{*} \times X \times \Sigma^{*}$ as follows: $x[\xi] y \succ x^{\prime}\left[\xi^{\prime}\right] y^{\prime}$ if (i) $\xi>\xi^{\prime}$, or (ii) $\xi=\xi^{\prime}$ and $x y \succ x^{\prime} y^{\prime}$ on $\Sigma^{*}$, or (iii) $\xi=\xi^{\prime}, x y=x^{\prime} y^{\prime}$ and $y \succ y^{\prime}$ on $\Sigma^{*}$.

The order $\succ$ can be extended to a partial order $\succ$ on $F \cdot X \cdot F$ in a similar manner as we did on $F$ in Section 2. An element $f$ of $F \cdot X \cdot F$ is uniquely written as

$$
f=\sum k_{i} x_{i}\left[\xi_{i}\right] y_{i}
$$

with $k_{i} \in K \backslash\{0\}, x_{i}, y_{i} \in \Sigma^{*}$ and $\xi_{i} \in X$, where $x_{i}\left[\xi_{i}\right] y_{i}$ are different elements in $\Sigma^{*} \times X \times \Sigma^{*}$. The leading term $\operatorname{lt}(f)$ of $f$ is the term $k_{i} x_{i}\left[\xi_{i}\right] y_{i}$ such that $x_{i}\left[\xi_{i}\right] y_{i} \succ x_{j}\left[\xi_{j}\right] y_{j}$ for all $j \neq i$. The element $f$ is monic if the coefficient $k_{i}$ of the leading term $k_{i} x_{i}\left[\xi_{i}\right] y_{i}$ is 1 . If, moreover, $x_{i}=1, f$ is called very monic.

A rewriting rule is a pair $(s, t)$ with $s \in \Sigma^{*} \times X \times \Sigma^{*}$ and $t \in F \cdot X \cdot F$ such that $s \succ t$. A rewriting system $T$ on $F \cdot X \cdot F$ is a set of rewriting rules. If $f \in F \cdot X \cdot F$ has a term $k \cdot x[\xi] y, x=x^{\prime} u, y=v y^{\prime}$ and $s=u[\xi] v \rightarrow t \in T$, then $f \rightarrow_{T} f-k \cdot x^{\prime}(u[\xi] v-t) y^{\prime}$ by an application of the rule $s \rightarrow t$.

A rule $u \rightarrow v(u-v \in G)$ in $R_{G}$ is also applied to a term $k \cdot x[\xi] y$ of $f$, if $x=x^{\prime} u x^{\prime \prime}$ or $y=y^{\prime} u y^{\prime \prime}$. In the former case, $f \rightarrow_{G} f-k \cdot x^{\prime}(u-v) x^{\prime \prime}[\xi] y$, and in the latter, $f \rightarrow_{G} f-k \cdot x[\xi] y^{\prime}(u-v) y^{\prime \prime}$. The relation $\rightarrow_{G}$ on $F \cdot X \cdot F$ is complete, because $\rightarrow_{G}$ is complete on $F$. So, any $f \in F \cdot X \cdot F$ has the unique normal form $\hat{f}$ with respect to $\rightarrow_{G}$. An element $f$ written as (3) is $G$-irreducible, if and only if every $x_{i}$ and $y_{i}$ are $G$-irreducible. Thus, we have $\hat{f}=\sum k_{i} \hat{x}_{i}\left[\xi_{i}\right] \hat{y}_{i}$.

Let $\rightarrow_{T, G}=\rightarrow_{T} \cup \rightarrow_{G}$, then $\rightarrow_{T, G}$ is a noetherian relation on $F \cdot X \cdot F$ because $f \rightarrow_{T, G} g$ implies $f \succ g$ by the compatibility of $\succ$. Let $\rightarrow_{T, G}^{*}$ and $\leftrightarrow_{T, G}^{*}$ be the reflexive transitive closure and the reflexive symmetric transitive closure of $\rightarrow_{T, G}$, respectively. An element $f \in F \cdot X \cdot F$ is $(T, G)$-irreducible, if no rule from $T \cup R_{G}$ is applied to $f$; otherwise, $f$ is $(T, G)$-reducible. Let $L(T, G)$ be the $F$-subbimodule of $F \cdot X \cdot F$ generated by $H_{T} \cup I \cdot X \cup X \cdot I$, where $H_{T}=\{s-t \mid s \rightarrow t \in T\}$. A similar result to Proposition 2.1 holds.

Proposition 3.1. The relation $\leftrightarrow_{T, G}^{*}$ is equal to the F-bimodule congruence of $F \cdot X \cdot F$ modulo $L(T, G) ; f \leftrightarrow_{T, G}^{*} g \Leftrightarrow f \equiv g(\bmod L(T, G))$. In particular, $L(T, G)=\left\{f \in F \cdot X \cdot F \mid f \leftrightarrow_{T, G}^{*} 0\right\}$.

Due to Proposition 3.1 the quotient $M=M(T, G)=F \cdot X \cdot F / \leftrightarrow_{T, G}^{*}$ is an $F$-bimodule equal to $F \cdot X \cdot F / L(T, G)$. But, moreover, $M$ is an $A$-bimodule in a natural way because $x \leftrightarrow_{G}^{*} y$ implies $x \cdot f \leftrightarrow_{T, G}^{*} y \cdot f$ and $f \cdot x \leftrightarrow_{T, G}^{*} f \cdot y$ for $x, y \in F$ and $f \in F \cdot X \cdot F$. Let $\eta: F \cdot X \cdot F \rightarrow M$ be the natural surjection which is a morphism of $F$-bimodules. Let $\rho_{X}: F \cdot X \cdot F \rightarrow A \cdot X \cdot A$ be the morphism of $K$-modules defined by

$$
\rho_{X}(x[\xi] y)=\rho(x)[\xi] \rho(y)
$$


for $x, y \in \Sigma^{*}$ and $\xi \in X$. Clearly, $\rho_{X}$ is a morphism of $F$-bimodules. Since $M$ is an $A$-bimodule, we have a surjection $\bar{\eta}: A \cdot X \cdot A \rightarrow M$ with the following commutative diagram:

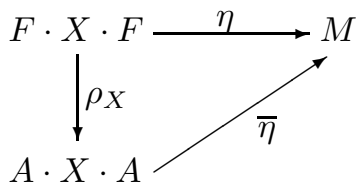

Hence, $\operatorname{Ker}(\bar{\eta})=\rho_{X}(L(T, G))$ is an $A$-subbimodule of $A \cdot X \cdot A$, which is denoted by $L_{A}(T) . L_{A}(T)$ is the $A$-subbimodule of $A \cdot X \cdot A$ generated by $\rho_{X}\left(H_{T}\right)$, and $M \cong(A \cdot X \cdot A) / L_{A}(T)$. Thus

Proposition 3.2. The quotient $M=M(T, G)=(F \cdot X \cdot F) / L(T, G)$ is an $A$ bimodule with the actions induced from the actions of $F$ to $F \cdot X \cdot F$. It is isomorphic to $(A \cdot X \cdot A) / L_{A}(T)$, where $L_{A}(T)$ is the A-subbimodule of $A \cdot X \cdot A$ generated by $\rho_{X}\left(H_{T}\right)=\left\{\rho_{X}(s-t) \mid s \rightarrow t \in T\right\}$.

If the rewriting system $\rightarrow_{T, G}$ is complete on $F \cdot X \cdot F$, we say $T$ is complete modulo $G$. Similar results to Proposition 2.3 hold in this situation.

Proposition 3.3. If a rewriting system $T$ on $F \cdot X \cdot F$ is complete modulo $G$, then for any $f \in F \cdot X \cdot F$, there is a unique $(T, G)$-irreducible element (the normal form of $f) \tilde{f} \in F \cdot X \cdot F$ such that $f \rightarrow_{T, G}^{*} \tilde{f}$, and for any $f, g \in F \cdot X \cdot F$ we have

$$
\tilde{f}=\tilde{g} \Leftrightarrow f \leftrightarrow_{T, G}^{*} g \Leftrightarrow f-g \rightarrow_{T, G}^{*} 0 \Leftrightarrow \eta(f)=\eta(g) .
$$

Obviously, the empty set $\emptyset$ is complete modulo $G$, and $\rightarrow \emptyset, G=\rightarrow_{G}$ and $\tilde{f}=\hat{f}$. Since $f \leftrightarrow_{G}^{*} g \Leftrightarrow \rho_{X}(f)=\rho_{X}(g)$ for $f, g \in F \cdot X \cdot F$, the morphism $\bar{\eta}: A \cdot X \cdot A \rightarrow M$ in (4) is an isomorphism in this case. Accordingly,

$$
\operatorname{Ker}\left(\rho_{X}\right)=L(\emptyset, G)=\{f \in F \cdot X \cdot F \mid \hat{f}=0\}=I \cdot X \cdot F+F \cdot X \cdot I
$$

is the $F$-subbimodule generated by $I \cdot X \cup X \cdot I$. Let $I_{X}$ denote this subbimodule. In general, $L(T, G)$ contains $I_{X}$.

A subset $H$ of $F \cdot X \cdot F$ is a Gröbner base (modulo $G$ ), if every element of $H$ is monic and the associated system $T_{H}=\{\operatorname{lt}(f) \rightarrow-\operatorname{rt}(f) \mid f \in H\}$ is a complete rewriting system on $F \cdot X \cdot F$ modulo $G$. For an $F$-subbimodule $L$ of $F \cdot X \cdot F$, if $H$ is a Gröbner base such that $L=L(H, G)=L\left(T_{H}, G\right), H$ is said to be a Gröbner base of $L$. It is also called a Gröbner base for the $A$-subbimodule $\rho_{X}(L)$ of $A \cdot X \cdot A$. We write $\rightarrow_{H, G}$ and $\rightarrow_{H, G}^{*}$ for $\rightarrow_{T_{H, G}}$ and $\rightarrow_{T_{H, G}^{*}}^{*}$ respectively. A $\left(\rightarrow_{H, G}\right)$-(ir)reducible element is called $(H, G)$-(ir)reducible. The quotient $M(H, G)=(F \cdot X \cdot F) / L(H, G)=(A \cdot X \cdot A) / L_{A}(H)$ is called the $A$-bimodule defined by a pair $(G, H)$ of Gröbner bases, where $L_{A}(H)=L_{A}\left(T_{H}\right)$.

Summarizing the above argument we have

Proposition 3.4. (1) The empty set $\emptyset$ is a Gröbner base of the F-subbimodule $I_{X}=I \cdot X \cdot F+F \cdot X \cdot I$ of $F \cdot X \cdot F$ modulo $G$, and $A \cdot X \cdot A$ is the A-bimodule defined by it. For $f, g \in F \cdot X \cdot F$ it holds that $\hat{f}=\hat{g} \Leftrightarrow \rho_{X}(f)=\rho_{X}(g)$.

(2) If $H$ is a Gröbner base modulo $G$ on $F \cdot X \cdot F$ of an $F$-subbibmodule $L$ of $F \cdot X \cdot F$, then $M(H, G)=F \cdot X \cdot F / L=A \cdot X \cdot A / L_{A}(H)$, any $f \in F \cdot X \cdot F$ has the unique normal form $\tilde{f}$ with respect to $T_{H}$, and $\tilde{f}=\tilde{g} \Leftrightarrow \eta(f)=\eta(g)$ in $M(H, G)$ for $f, g \in F \cdot X \cdot F$. 


\section{PROPER CRITICAL PAIRS AND STANDARD REDUCTIONS}

$G$ is continued to be a normalized Gröbner base on the free algebra $F$ over $K$. Let $T$ be a rewriting system on $F \cdot X \cdot F$ and let $H=H_{T}=\{s-t \mid s \rightarrow t \in T\}$. We shall consider critical pairs for $T$ modulo $G$. To simplify the argument we consider only a special type of systems which we need in this paper. We say $T$ (and $H$ ) is very monic if the left-hand side of each rule of $T$ is very monic. Moreover, we can normalize $T$ as we did for rewriting systems on $F$ in Section 3. $T$ is normalized modulo $G$ if for any $s \rightarrow t \in T, t$ is $(H, G)$-irreducible and $s$ is $(H \backslash\{s-t\}, G)$-irreducible. We have a similar result to Proposition 2.9, a proof of which is omitted.

Proposition 4.1. If an F-subbimodule L has a Gröbner base $H$ modulo $G$, it has a normalized Gröbner base $H^{\prime}$ modulo $G$. If $H$ is finite (resp. very monic), we can choose $H^{\prime}$ as finite (resp. very monic).

From now on $T$ is a normalized very monic rewriting system on $F \cdot X \cdot F$. Let $[\xi] x \rightarrow t \in T\left(t \in F \cdot X \cdot F\right.$ and $\left.\xi \in X, x \in \Sigma^{*}\right)$ and $u-v \in G$. Suppose that $x$ overlaps with $u$, that is, $x=x^{\prime} z, u=z u^{\prime}$ with $z \neq 1$. We can apply the rules on $[\xi] x u^{\prime}=[\xi] x^{\prime} u$ in two ways, and obtain a critical pair

$$
\left([\xi] x u^{\prime} \rightarrow_{T} t u^{\prime},[\xi] x^{\prime} u \rightarrow_{G}[\xi] x^{\prime} v\right)
$$

of reductions and a critical pair $\left(t u^{\prime},[\xi] x^{\prime} v\right)$ of elements. The critical pair is resolvable if $t u^{\prime} \downarrow_{T, G}[\xi] x^{\prime} v$, that is, there is $f \in F \cdot X \cdot F$ such that $t u^{\prime} \rightarrow_{T, G}^{*} f$ and $[\xi] x^{\prime} v \rightarrow T_{T, G}^{*} f$. The critical pair is proper if $x u^{\prime}$ is a minimal $G$-reducible word, that is, any proper prefix of $x u^{\prime}$ is $G$-irreducible. For the (proper) critical pair $\left(t u^{\prime},[\xi] x^{\prime} v\right)$ above, the difference $t u^{\prime}-[\xi] x^{\prime} v$ is a (proper) $S$-polynomial.

Proposition 4.2. A normalized very monic system $T$ on $F \cdot X \cdot F$ is complete modulo $G$ if all the proper critical pairs are resolvable.

Proof. As in the proof of Proposition 2.8, it suffices to show that, under the condition that all the proper critical pairs are resolvable, $T$ is locally confluent. Again we shall prove that $f \downarrow_{T, G} g$ holds for any $f, g, h \in F \cdot X \cdot F$ such that $h \rightarrow_{T, G} f$ and $h \rightarrow_{T, G} g$ by induction on $h$. For the same reason discussed in the proof of Proposition 2.8, we may suppose that $h=k x[\xi] y+h^{\dagger}$, where $k \in K \backslash\{0\}, x, y \in \Sigma^{*}, \xi \in X$ and $h^{\dagger}$ is an irreducible element of $F \cdot X \cdot F$, and two reductions $h \rightarrow_{T, G} f$ and

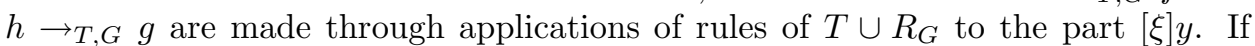
both are made through applications of rules from $G, f \downarrow_{G} g$ holds because $R_{G}$ is complete. Since $T$ is very monic and normalized, two different applications of rules from $T$ to $[\xi] y$ is impossible.

Thus, we may suppose that $y=y^{\prime} y^{\prime \prime}=y_{1} u y_{2},[\xi] y^{\prime} \rightarrow t \in T, u-v \in G$ with $y^{\prime}, y^{\prime \prime}, y_{1}, y_{2} \in \Sigma^{*}$, and $f=k \cdot x t y^{\prime \prime}+h^{\dagger}, g=k \cdot x[\xi] y_{1} v y_{2}+h^{\dagger}$. If $y^{\prime}$ and $u$ are disjoint in $y$, that is, $y_{1}=y^{\prime} z$ for some $z \in \Sigma^{*}$, then $f=k \cdot x t z u y_{2}+h^{\dagger} \rightarrow_{G} k \cdot x t z v y_{2}+h^{\dagger}$, $g=k \cdot x[\xi] y^{\prime} z v y_{2}+h^{\dagger} \rightarrow_{T} k \cdot x t z v y_{2}+h^{\dagger}$, and hence $f \downarrow_{T, G} g$ holds. If they overlap, that is, $y^{\prime}=y_{1} z$, with nonempty prefix $z$ of $u ; u=z w$, then we have a critical pair $\left([\xi] y^{\prime} w \rightarrow_{T} t \cdot w,[\xi] y_{1} u \rightarrow_{G}[\xi] y_{1} v\right)$ of reductions. If this critical pair is proper, then it is resolved by assumption and hence $f \downarrow_{T, G} g$ holds.

If it is not proper, $y$ has a subword $u^{\prime}$ with $y^{\prime}=y_{3} z^{\prime}, y^{\prime \prime}=w^{\prime} y_{4}$ and $u^{\prime}=z^{\prime} w^{\prime}$, such that $u^{\prime}-v^{\prime} \in G$ and $\left([\xi] y^{\prime} w^{\prime} \rightarrow_{T} t \cdot w^{\prime},[\xi] y_{3} u^{\prime} \rightarrow_{G}[\xi] y_{3} v^{\prime}\right)$ is a proper critical pair of reductions. Since it is resolved by assumption, $t \cdot w^{\prime} \rightarrow_{T, G}^{*} h^{\prime}$ and $[\xi] y_{3} v^{\prime} \rightarrow_{T, G}^{*} h^{\prime}$ for some $h^{\prime} \in F \cdot X \cdot F$. Therefore, $f=k \cdot x t y^{\prime \prime}+h^{\dagger}=k \cdot x t w^{\prime} y_{4}+$ 
$h^{\dagger} \rightarrow_{T, G}^{*} f^{\prime}$ and $k \cdot x[\xi] y_{3} v^{\prime} y_{4}+h^{\dagger} \rightarrow_{T, G}^{*} f^{\prime}$, where $f^{\prime}=k \cdot x h^{\prime} y_{4}+h^{\dagger}$. On the other hand, since $h \rightarrow_{G} g, h \rightarrow_{G} k \cdot x[\xi] y_{3} v^{\prime} y_{4}+h^{\dagger}$ and $T_{G}$ is complete, $g \rightarrow_{G}^{*} g^{\prime}$ and $k \cdot x[\xi] y_{3} v^{\prime} y_{4}+h^{\dagger} \rightarrow_{G} g^{\prime}$ for some $g^{\prime} \in F \cdot X \cdot F$. Moreover, $f^{\prime}$ and $g^{\prime}$ have the common ascendant $k \cdot x[\xi] y_{3} v^{\prime} y_{4}+h^{\dagger} \prec h$. Hence, by the induction hypothesis (the same as (i) in the proof of Proposition 2.8 holds), we have $f^{\prime} \downarrow_{T, G} g^{\prime}$, and consequently, we find that $f \downarrow_{T, G} g$.

Proposition 4.2 gives the following elaborated version of Proposition 2.9 for rewriting systems on bimodules.

Proposition 4.3. For a normalized very monic rewriting system $T$ on $F \cdot X \cdot F$ the following statements are equivalent.

(1) $T$ is complete modulo $G$.

(2) Every critical pair is resolvable.

$\left(2^{\prime}\right)$ Every proper critical pair is resolvable.

(3) Every S-polynomial is reduced to 0.

$\left(3^{\prime}\right)$ Every proper $S$-polynomial is reduced to 0.

(4) Every element in $L(T, G)$ is reduced to 0.

In calculating reduction sequences in $F \cdot X \cdot F$ we have to be careful that a rule from $T \cup R_{G}$ must be applied to a whole term $k \cdot x[\xi] y(k \in K \backslash\{0\})$ of an element of $F \cdot X \cdot F$ not to a part $k^{\prime} \cdot x[\xi] y\left(k^{\prime}=k-k^{\prime \prime}, k^{\prime \prime} \in K \backslash\{0\}\right)$ of it in each reduction step. Accordingly, for $f, g, f^{\prime} g^{\prime} \in F \cdot X \cdot F$ the reductions $f \rightarrow_{T, G}^{*} f^{\prime}$ and $g \rightarrow_{T, G}^{*} g^{\prime}$ do not guarantee the reduction $f+g \rightarrow_{T, G}^{*} f^{\prime}+g^{\prime}$, even if $f^{\prime}$ and $g^{\prime}$ are $(T, G)$-irreducible. In this sense the reduction in $F \cdot X \cdot F$ is not additive, and this causes some difficulty in our calculation. Fortunately, considering a special type of reduction we can avoid this difficulty.

A reduction

$$
f_{1} \rightarrow_{T, G} f_{2} \rightarrow_{T, G} \cdots \rightarrow_{T, G} f_{n}
$$

is called standard, if for every $i=1, \ldots, n-1$,

(i) when $f_{i}$ is $G$-reducible, the reduction $f_{i} \rightarrow_{T, G} f_{i+1}$ is made through an application of a rule from $G$, and

(ii) when $f_{i}$ is $G$-irreducible, a rule from $T$ is applied to the smallest $T$-reducible term of $f_{i}$ with respect to $\succ$ in the reduction step $f_{i} \rightarrow_{T, G} f_{i+1}$.

If $f_{1}$ is reduced to $f_{n}$ through a standard reduction as above, we write it as $f_{1} \Rightarrow_{T, G}^{*} f_{n}$. A standard one-step reduction by a rule from $T$ is denoted by $\Rightarrow_{T}$, that is, $f \Rightarrow_{T} g$ if $f$ is $G$-irreducible and $g$ is obtained by applying a rule of $T$ to the smallest $T$-reducible term of $f$.

Since $\rightarrow_{G}$ is complete, the standard reduction (5) can be rewritten as

$$
f_{1}=g_{1} \rightarrow_{G}^{*} \hat{g}_{1} \Rightarrow_{T} g_{2} \rightarrow_{G}^{*} \hat{g}_{2} \Rightarrow_{T} \cdots \Rightarrow_{T} g_{m} \rightarrow_{G} \hat{g}_{m}=f_{n} .
$$

Since $T$ is very monic and normalized, in the step $\hat{g}_{i} \Rightarrow_{T} g_{i+1}$ in the above reduction sequence, only one rule from $T$ is applicable to the smallest $T$-reducible term of $\hat{g}_{i}$. In this sense, a standard reduction from $f$ to a $(T, G)$-irreducible element is unique. Thus we have

Lemma 4.4. For any $f \in F \cdot X \cdot F$, there is a unique $(T, G)$-irreducible element $f^{\prime} \in F \cdot X \cdot F$ such that $f \Rightarrow_{T, G}^{*} f^{\prime}$. If $T$ is complete modulo $G$, this $f^{\prime}$ is equal to the normal form $\tilde{f}$ of $f$. 
The additivity of the standard reduction is stated in the following lemma, which will be extensively used in our calculations later. We abbreviate $\Rightarrow_{T, G}^{*}$ to $\Rightarrow^{*}$.

Lemma 4.5. If $f \Rightarrow^{*} f^{\prime}$ and $g \Rightarrow^{*} g^{\prime}$ for $f, f^{\prime}, g, g^{\prime} \in F \cdot X \cdot F$ such that $f^{\prime}$ and $g^{\prime}$ are $(T, G)$-irreducible, then $k \cdot f+\ell \cdot g \Rightarrow^{*} k \cdot f^{\prime}+\ell \cdot g^{\prime}$ for any $k, \ell \in K$.

Proof. Because $f \Rightarrow^{*} f^{\prime}$ implies $k \cdot f \Rightarrow^{*} k \cdot f^{\prime}$ for any $k \in K$, it suffices to prove the additivity $f+g \Rightarrow^{*} f^{\prime}+g^{\prime}$. We shall prove this by induction on the pair $(f, g)$ with respect to $\succ$. If $f=0$ or $g=0$, the assertion trivially holds. So suppose that $f$ and $g$ are nonzero. We have $(f+g)=\hat{f}+\hat{g}$ because $G$ is complete (Corollary 2.4), and $\hat{f} \Rightarrow^{*} f^{\prime}$ and $\hat{g} \Rightarrow^{*} g^{\prime}$ by the definition of standard reduction. If $f$ or $g$ is $G$ reducible, $f \succ \hat{f}$ or $g \succ \hat{g}$ holds, and by the induction hypothesis, $\hat{f}+\hat{g} \Rightarrow^{*} f^{\prime}+g^{\prime}$. Hence $f+g \rightarrow_{G}^{*}\left(f+g \hat{)}=\hat{f}+\hat{g} \Rightarrow^{*} f^{\prime}+g^{\prime}\right.$.

Suppose $f$ and $g$ are $G$-irreducible, but $f$ is $T$-reducible. Let $k_{1} \cdot f_{1}$ with $k_{1} \in$ $K \backslash\{0\}$ and $f_{1} \in \Sigma^{*} \times X \times \Sigma^{*}$ be the smallest $T$-reducible term of $f$. Then, $f_{1} \Rightarrow_{T} f_{1}^{\prime}$, $f \Rightarrow_{T} k_{1} \cdot f_{1}^{\prime}+f_{2}$ and $k_{1} \cdot f_{1}^{\prime}+f_{2} \Rightarrow^{*} f^{\prime}$, where $f_{2}=f-k_{1} \cdot f_{1}$. If, here, $g$ is $T$-irreducible, then $g=g^{\prime}, f+g \Rightarrow_{T} k_{1} \cdot f_{1}^{\prime}+f_{2}+g$, and by the induction hypothesis, $\left(k_{1} \cdot f_{1}^{\prime}+f_{2}\right)+g \Rightarrow^{*} f^{\prime}+g$. Consequently, $f+g \Rightarrow^{*} f^{\prime}+g^{\prime}$.

Next, suppose $g$ is also $T$-reducible and let $\ell_{1} \cdot g_{1}$ with $\ell_{1} \in K \backslash\{0\}$ and let $g_{1} \in \Sigma^{*} \times X \times \Sigma^{*}$ be the smallest $T$-reducible term of $g$. As above $g_{1} \Rightarrow_{T} g_{1}^{\prime}$ and $\ell_{1} \cdot g_{1}^{\prime}+g_{2} \Rightarrow^{*} g^{\prime}$, where $g_{2}=g-\ell_{1} \cdot g_{1}$. If $f_{1}=g_{1}$ and $k_{1}+\ell_{1} \neq 0$, then $f+g \Rightarrow_{T}$ $\left(k_{1}+\ell_{1}\right) \cdot f_{1}^{\prime}+f_{2}+g_{2}$, and $\left(k_{1}+\ell_{1}\right) \cdot f_{1}^{\prime}+f_{2}+g_{2}=\left(k_{1} \cdot f_{1}^{\prime}+f_{2}\right)+\left(\ell_{1} \cdot g_{1}^{\prime}+g_{2}\right) \Rightarrow^{*} f^{\prime}+g^{\prime}$ by the induction hypothesis. If $f_{1}=g_{1}$ and $k_{1}+\ell_{1}=0$, then $f+g=f_{2}+g_{2}=$ $\left(k_{1} \cdot f_{1}^{\prime}+f_{2}\right)+\left(\ell_{1} \cdot g_{1}^{\prime}+g_{2}\right) \Rightarrow^{*} f^{\prime}+g^{\prime}$ by the induction hypothesis. If $f_{1} \prec g_{1}$, then $k_{1} f_{1}$ is the smallest $T$-reducible term of $f+g$ and hence $f+g \Rightarrow_{T} k_{1} \cdot f_{1}^{\prime}+f_{2}+g$. The last element is reduced to $f^{\prime}+g^{\prime}$ through standard reduction by the induction hypothesis. Finally, if $f_{1} \succ g_{1}$, then $f+g \Rightarrow_{T} f+\ell_{1} g_{1}^{\prime}+g_{2} \Rightarrow^{*} f^{\prime}+g^{\prime}$. In every possible case we have shown that $f+g \Rightarrow^{*} f^{\prime}+g^{\prime}$.

The following will also be used in the next section.

Lemma 4.6. Let $f, f^{\prime} \in F \cdot X \cdot F$ and $x \in \Sigma^{*}$. If $f \Rightarrow^{*} f^{\prime}$ and $f^{\prime}$ is $(T, G)$ irreducible, then $x \cdot f \Rightarrow^{*}\left(x f^{\prime}\right)$.

Proof. By $K$-linearity of standard reduction and the mapping ^, we may suppose that $f$ is a monic monomial, that is, $f=y[\xi] z$ with $\xi \in X$ and $y, z \in \Sigma^{*}$. We proceed by induction on $f$ with respect to $\succ$. If $f$ is $G$-reducible, $f \succ \hat{f}$ and $\hat{f} \Rightarrow^{*} f^{\prime}$. By the induction hypothesis $x \cdot \hat{f} \Rightarrow^{*}\left(x f^{\prime}\right)$, and thus, $x \cdot f \rightarrow_{G}^{*} x \cdot \hat{f} \Rightarrow^{*}\left(x f^{\prime}\right)$. If $f$ is $(T, G)$-irreducible, then $f=f^{\prime}$ and the assertion holds. So assume that $y$ and $z$ are $G$-irreducible but $f$ is $T$-reducible. Then, $z=z_{1} z_{2}$ and $[\xi] z_{1} \rightarrow t \in T$, $f \Rightarrow_{T} y \cdot t \cdot z_{2}$ and $y \cdot t \cdot z_{2} \Rightarrow^{*} f^{\prime}$. Because all the terms in $y \cdot t \cdot z_{2}$ are less than $f$, we see $x\left(y \cdot t \cdot z_{2}\right) \rightarrow_{G}^{*}(x y) \cdot t \cdot z_{2} \Rightarrow^{*}\left(x f^{\prime}\right)$ by the induction hypothesis. Thus, we have the desired standard reduction

$$
x \cdot f \rightarrow_{G}^{*}(x y) \cdot[\xi] \cdot z \Rightarrow_{T}(x y) \cdot t \cdot z_{2} \Rightarrow^{*}\left(x f^{\prime} \hat{)} .\right.
$$

\section{Gröbner bases made from CRitical pairs of Reductions}

As in Section 4, let $G$ be a normalized Gröbner base of an ideal $I$ of the free algebra $F$, and let $\rho: F \rightarrow A=F / I$ be the surjection. Let $H$ be a normalized very monic Gröbner base modulo $G$ of an $F$-subbimodule $L$ of $F \cdot X \cdot F$, and let 
$M=F \cdot X \cdot F / L$ be the $A$-bimodule defined by $(H, G)$. We consider the free $F$ bimodule $F \cdot H \cdot F$ generated by the set $H$. For $h \in H$, $[h]$ denotes the formal generator of $F \cdot H \cdot F$ corresponding to $h \in H$. Let $\delta: F \cdot H \cdot F \rightarrow F \cdot X \cdot F$ be a morphism of $F$-bimodules defined by

$$
\delta([h])=h .
$$

We have $L=L(H, G)=\operatorname{Im}(\delta)+I_{X}$. In this section we shall construct a Gröbner base on $F \cdot H \cdot F$, and in the next section we shall show that it is actually a Gröbner base of $\operatorname{Ker}(\delta)+I_{H}$, where $I_{H}=I \cdot H \cdot F+F \cdot H \cdot I$.

We need to introduce an order $\succ$ on $\Sigma^{*} \times H \times \Sigma^{*}$ under the condition that a compatible well-order $\succ$ is already given on $\Sigma^{*} \times X \times \Sigma^{*}$. For $f=x[h] y$ and $g=x^{\prime}\left[h^{\prime}\right] y^{\prime} \in \Sigma^{*} \times H \times \Sigma^{*}$ with $x, y, x^{\prime}, y^{\prime} \in \Sigma^{*}$ and $h, h^{\prime} \in H, x \cdot \operatorname{lt}(h) \cdot y$ and $x^{\prime} \cdot \operatorname{lt}\left(h^{\prime}\right) \cdot y^{\prime}$ are considered to be elements of $\Sigma^{*} \times X \times \Sigma^{*}$. Define $f \succ g$ if and only if

(i) $x \cdot \operatorname{lt}(h) \cdot y \succ x^{\prime} \cdot \operatorname{lt}\left(h^{\prime}\right) \cdot y^{\prime}$ in $\Sigma^{*} \times X \times \Sigma^{*}$, or

(ii) $x \cdot \operatorname{lt}(h) \cdot y=x^{\prime} \cdot \operatorname{lt}\left(h^{\prime}\right) \cdot y^{\prime}$ and $|y|>\left|y^{\prime}\right|$, or

(iii) $x \cdot \operatorname{lt}(h) \cdot y=x^{\prime} \cdot \operatorname{lt}\left(h^{\prime}\right) \cdot y^{\prime},|y|=\left|y^{\prime}\right|$ and $|x|<\left|x^{\prime}\right|$.

This $\succ$ is a total order. In fact, if $x \cdot \operatorname{lt}(h) \cdot y=x^{\prime} \cdot \operatorname{lt}\left(h^{\prime}\right) \cdot y^{\prime},|y|=\left|y^{\prime}\right|$ and $|x|=\left|x^{\prime}\right|$, then $\operatorname{lt}(h)=\operatorname{lt}\left(h^{\prime}\right)$ and hence $h=h^{\prime}$ because $H$ is normalized. Moreover, $\succ$ is a compatible well-order on $\Sigma^{*} \times H \times \Sigma^{*}$ because it is defined through the compatible well-order on $\Sigma^{*} \cdot X \cdot \Sigma^{*}$. It is extended to the partial order $\succ$ on $F \cdot H \cdot F$ as before.

Now, we define a mapping $\beta$ from $F \cdot X \cdot F$ to $F \cdot H \cdot F$, which will play an important role in the rest of the paper. For an element $f=x \cdot[\xi] \cdot y \in \Sigma^{*} \times X \times \Sigma^{*}$ we define an element $\beta(f)$ of $F \cdot H \cdot F$ by induction with respect to $\succ$. First, if $f$ is $(G \cup H)$-irreducible, let $\beta(f)=0$. Next, if $f$ is $G$-reducible, let $\beta(f)=\beta(\hat{f})=$ $\beta(\hat{x}[\xi] \hat{y})$. Finally, suppose $f$ is $G$-irreducible but $H$-reducible, that is, $y=y^{\prime} y^{\prime \prime}$ and $h=[\xi] y^{\prime}-t \in H$. Because $H$ is normalized, this $h$ is unique. Because $f \succ x t y^{\prime \prime}$, $x t y^{\prime \prime}$ is written as $\sum k_{i} x_{i}\left[\xi_{i}\right] y_{i}$ with $x_{i}\left[\xi_{i}\right] y_{i} \prec f$. Hence every $\beta\left(x_{i}\left[\xi_{i}\right] y_{i}\right)$ is already defined by the induction hypothesis. Now, define

$$
\beta(f)=x[h] y^{\prime \prime}+\sum k_{i} \beta\left(x_{i}\left[\xi_{i}\right] y_{i}\right) .
$$

Moreover, for an element $f$ of $F \cdot X \cdot F$ expressed as (3) define

$$
\beta(f)=\sum k_{i} \beta\left(x_{i}\left[\xi_{i}\right] y_{i}\right) .
$$

By the definition we easily see

Lemma 5.1. (1) $\beta$ is a morphism of $K$-modules, that is, $\beta(k f+\ell g)=k \cdot \beta(f)+$ $\ell \cdot \beta(g)$ for $k, \ell \in K$ and $f, g \in F \cdot X \cdot F$.

(2) $\beta(f)=\beta(\hat{f})$ for $f \in F \cdot X \cdot F$, where $\hat{f}$ is the normal form of $f$ with respect to $G$.

(3) $\beta(f)$ is $G$-irreducible for $f \in F \cdot X \cdot F$.

(4) $\beta(x \cdot f)=\left(x \cdot \beta(f) \hat{)}\right.$ for $x \in \Sigma^{*}$ and $f \in F \cdot X \cdot F$.

Let $h=[\xi] x-t \in H$ and $u^{\prime \prime} \in \Sigma^{*}$ such that $x=x^{\prime} u^{\prime}, u=u^{\prime} u^{\prime \prime}, u-v \in G$ and $x u^{\prime \prime}$ is a minimal $G$-reducible word. We have a proper critical pair $\left([\xi] x u^{\prime \prime} \rightarrow_{H} t u^{\prime \prime}\right.$, $\left.[\xi] x^{\prime} u \rightarrow_{G}[\xi] x^{\prime} v\right)$ of reductions and a proper $S$-polynomial $p=t u^{\prime \prime}-[\xi] x^{\prime} v$. We consider an element $c$ of $F \cdot H \cdot F$ corresponding to this critical pair defined by

$$
c=[h] u^{\prime \prime}+\beta(p)=[h] u^{\prime \prime}+\beta\left(t u^{\prime \prime}\right)-\beta\left([\xi] x^{\prime} v\right) .
$$


Since $[\xi] x u^{\prime \prime} \succ t u^{\prime \prime}$ and $[\xi] x u^{\prime \prime} \succ[\xi] x^{\prime} v$, we see $[h] u^{\prime \prime} \succ \beta\left(t u^{\prime \prime}\right)-\beta\left([\xi] x^{\prime} v\right)$ by the definitions of $\beta$ and our order $\succ$ on $\Sigma^{*} \times H \times \Sigma^{*}$, that is, ht $(c)=[h] u^{\prime \prime}$. In particular, $c$ is very monic.

Let $C$ be the set of the elements $c$ given as (6) for all proper critical pairs of reductions. We have the rewriting system $T_{C}$ on $F \cdot H \cdot F$ associated with $C$, which is the set of all rules

$$
[h] u^{\prime \prime} \rightarrow \beta\left([\xi] x^{\prime} v\right)-\beta\left(t u^{\prime \prime}\right)
$$

corresponding to proper critical pairs.

As stated in Lemma $5.1,(3), \beta(f)$ is $G$-irreducible for $f \in F \cdot X \cdot F$. Moreover, it is also $C$-irreducible.

Lemma 5.2. For any $f \in F \cdot X \cdot F, \beta(f)$ is $(C, G)$-irreducible.

Proof. By Lemma 5.1, (1) and (2), it suffices to show the assertion for a $G$ irreducible monic monomial $f=x[\xi] y(x, y \in \operatorname{Irr}(G), \xi \in X)$. Since $\beta(f)=0$ if $f$ is $H$-irreducible, we may suppose that $f$ is $H$-reducible. Let $y=y^{\prime} y^{\prime \prime}$, $h=[\xi] y^{\prime}-t \in H$. Then, $\beta(f)=x \cdot[h] \cdot y^{\prime \prime}+\beta\left(x \cdot t \cdot y^{\prime \prime}\right)$ and $f \succ x \cdot t \cdot y^{\prime \prime}$. By the induction hypothesis $\beta\left(x \cdot t \cdot y^{\prime \prime}\right)$ is $(C, G)$-irreducible. Assume that $x \cdot[h] \cdot y^{\prime \prime}$ is $C$-reducible, that is, $y^{\prime \prime}=y_{1} y_{2}$ and $[h] y_{1}-s \in C$. So, we have a proper critical pair $\left([\xi] y^{\prime} y_{1} \rightarrow t \cdot y_{1},[\xi] y_{1}^{\prime} y_{2}^{\prime} y_{1} \rightarrow[\xi] y_{1}^{\prime} v^{\prime}\right)$ of reductions, where $y^{\prime}=y_{1}^{\prime} y_{2}^{\prime}$ and $y_{2}^{\prime} y_{1}-v^{\prime} \in G$. But this means that $y=y_{1}^{\prime} y_{2}^{\prime} y_{1} y_{2}$ is $G$-reducible, a contradiction.

Now we shall prove one of the key results for our construction that $C$ is a Gröbner base on $F \cdot H \cdot F$. To this end we need the following technical lemma.

Lemma 5.3. For $f \in F \cdot X \cdot F$ and $x \in F$ we have a standard reduction

$$
\beta(f) \cdot x \Rightarrow_{C, G}^{*} \beta(f \cdot x)-\beta(\tilde{f} \cdot x),
$$

where $\tilde{f}$ is the normal form of $f$ with respect to $G \cup H$.

Proof. We prove the assertion by induction on $f \cdot x$ with respect to $\succ$. Since the right-hand side of (7) is $(C, G)$-irreducible by Lemma 5.2 and the mappings $\beta$ and $\sim$ are $K$-linear, Lemma 4.5 tells us that we may suppose that $f$ is a monic monomial, that is, $f=w[\xi] y$ with $\xi \in X$ and $w, y \in \Sigma^{*}$. Moreover, due to Lemma 4.6 and Lemma 5.1, (2) and (4) we may suppose that $w=1$ and $y \in \operatorname{Irr}(G)$.

If $f$ is $H$-irreducible, then $f=\tilde{f}$ and $\beta(f)=0$, and hence both sides of (7) are zero. So, suppose that $f$ is $H$-reducible, that is, $y=y^{\prime} y^{\prime \prime}$ and $h=[\xi] y^{\prime}-t \in H$. By the definition of $\beta$ we have

$$
\beta(f)=[h] y^{\prime \prime}+\beta\left(t \cdot y^{\prime \prime}\right), \quad[h] y^{\prime \prime} \succ \beta\left(t \cdot y^{\prime \prime}\right) .
$$

Since $t \cdot y^{\prime \prime} \prec f$, by the induction hypothesis we have

$$
\beta\left(t \cdot y^{\prime \prime}\right) \cdot x \Rightarrow^{*} \beta\left(t \cdot y^{\prime \prime} x\right)-\beta\left(\left(t \cdot y^{\prime \prime}\right) \cdot x\right)=\beta\left(t \cdot y^{\prime \prime} x\right)-\beta(\tilde{f} \cdot x),
$$

where $\Rightarrow^{*}$ is the abbreviation of the standard reduction $\Rightarrow_{C, G}^{*}$. Thus, to show (7) it suffices to show

$$
[h] y^{\prime \prime} x \Rightarrow^{*} \beta([\xi] y x)-\beta\left(t \cdot y^{\prime \prime} x\right),
$$

again by the additivity of standard reduction in Lemma 4.5 .

If $y x$ is $G$-irreducible, we have $\beta([\xi] y x)=[h] y^{\prime \prime} x+\beta\left(t \cdot y^{\prime \prime} x\right)$, and we find that both sides of (8) are equal. So, we suppose that $y x=y^{\prime} y^{\prime \prime} x$ is $G$-reducible. We need to consider two cases,

(i) $y^{\prime \prime}=y_{1} y_{2}, y_{2} \neq 1, x=x_{1} x_{2}, y_{2} x_{1}-v \in G$, and 
(ii) $y^{\prime \prime} x$ is $G$-irreducible and $y^{\prime}=y_{1} y_{2}, y_{2} \neq 1, x=x_{1} x_{2}, y_{2} y^{\prime \prime} x_{1}-v \in G$.

In case (i) we have

$$
\begin{gathered}
{[\xi] y x \rightarrow_{G}[\xi] y^{\prime} y_{1} v x_{2} \rightarrow_{G}^{*}[\xi] y^{\prime} z,} \\
t \cdot y^{\prime \prime} x \rightarrow_{G}^{*} t \cdot z,
\end{gathered}
$$

and

$$
[h] y^{\prime \prime} x \rightarrow_{G}^{*}[h] z,
$$

where $z=\left(y^{\prime \prime} x\right)$. By Lemma 5.1, (2) we have

$$
\beta\left([\xi] y^{\prime} z\right)=\beta([\xi] y x), \beta(t \cdot z)=\beta\left(t \cdot y^{\prime \prime} x\right)
$$

Here, if $y^{\prime} z$ is $G$-irreducible, we have

$$
\beta\left([\xi] y^{\prime} z\right)=[h] z+\beta(t \cdot z) .
$$

This together with (10) shows that the right-hand side of (9) is equal to $\beta([\xi] y x)-$ $\beta\left(t \cdot y^{\prime \prime} x\right)$, as desired.

If $y^{\prime} z$ is $G$-reducible, then there is $u^{\prime}-v^{\prime} \in G$ such that $u^{\prime}=y_{4} z_{1}, y^{\prime}=y_{3} y_{4}$, $z=z_{1} z_{2}$. Here, we can choose the element $u^{\prime}-v^{\prime}$ of $G$ so that $y^{\prime} z_{1}$ is a minimal $G$-reducible word, that is, $\left([\xi] y^{\prime} z_{1} \rightarrow_{H} t z_{1},[\xi] y^{\prime} z_{1} \rightarrow_{G}[\xi] y_{3} v^{\prime}\right)$ is a proper critical pair of reductions. Thus, we have the rule

$$
[h] z_{1} \rightarrow \beta\left([\xi] y_{3} v^{\prime}\right)-\beta\left(t \cdot z_{1}\right)
$$

in $T_{C}$, and hence

$$
[h] z=[h] z_{1} z_{2} \Rightarrow_{C} \beta\left([\xi] y_{3} v^{\prime}\right) z_{2}-\beta\left(t \cdot z_{1}\right) z_{2},
$$

where $\Rightarrow_{C}$ denotes a one-step standard $C$-reduction. Since $f \cdot x \succ[\xi] y_{3} v^{\prime}$ and $f \cdot x \succ t z_{1} z_{2}$, by the induction hypothesis we have

$$
\beta\left([\xi] y_{3} v^{\prime}\right) z_{2} \Rightarrow^{*} \beta\left([\xi] y_{3} v^{\prime} z_{2}\right)-\beta\left(\left([\xi] y_{3} v^{\prime}\right) z_{2}\right)
$$

and

$$
\beta\left(t \cdot z_{1}\right) z_{2} \Rightarrow^{*} \beta\left(t \cdot z_{1} z_{2}\right)-\beta\left(\left(t \cdot z_{1}\right) z_{2}\right) .
$$

Since $\left([\xi] y_{3} v^{\prime}\right)=\left(t \cdot z_{1}\right)$ by the confluence of $\rightarrow_{H, G}$, we see

$$
[h] z \Rightarrow^{*} \beta\left([\xi] y_{3} v^{\prime} z_{2}\right)-\beta(t \cdot z)=\beta([\xi] y x)-\beta\left(t \cdot y^{\prime \prime} x\right) .
$$

(9) and (11) yield the desired reduction (8).

In case (ii), again we may suppose that $y x_{1}$ is a minimal $G$-reducible word, and hence we have the rule

$$
[h] y^{\prime \prime} x_{1} \rightarrow \beta\left([\xi] y_{1} v\right)-\beta\left(t \cdot y^{\prime \prime} x_{1}\right)
$$

in $T_{C}$. Since $y^{\prime \prime} x_{1} x_{2}=y^{\prime \prime} x$ is $G$-irreducible,

$$
[h] y^{\prime \prime} x \Rightarrow_{C} \beta\left([\xi] y_{1} v\right) \cdot x_{2}-\beta\left(t \cdot y^{\prime \prime} x_{1}\right) \cdot x_{2} .
$$

By the induction hypothesis,

$$
\beta\left([\xi] y_{1} v\right) \cdot x_{2} \Rightarrow^{*} \beta\left([\xi] y_{1} v x_{2}\right)-\beta\left(\left([\xi] y_{1} v\right) x_{2}\right)
$$

and

$$
\beta\left(t \cdot y^{\prime \prime} x_{1}\right) \cdot x_{2} \Rightarrow^{*} \beta\left(t \cdot y^{\prime \prime} x\right)-\beta\left(\left(t \cdot y^{\prime \prime} x_{1}\right) x_{2}\right) .
$$

Since $\left(t \cdot y^{\prime \prime} x_{1}\right)=\left([\xi] y_{1} v\right)$ and $\beta\left([\xi] y_{1} v x_{2}\right)=\beta([\xi] y x)$, we get the reduction (8) from (12) using Lemma 4.5.

Theorem 5.4. $C$ is a normalized very monic Gröbner base on $F \cdot H \cdot F$. 
Proof. We already know that $C$ is very monic. Let $c=[h] u^{\prime \prime}+\beta\left(t \cdot u^{\prime \prime}\right)-\beta\left([\xi] x^{\prime} v\right)$ be an element of $C$ given as (6), where $h=[\xi] x-t \in H, x=x^{\prime} u^{\prime}, u=u^{\prime} u^{\prime \prime}, u-v \in G$ and $x u^{\prime \prime}$ is a minimal $G$-reducible word. Lemma 5.2 tells us that $\beta\left(t u^{\prime \prime}\right)-\beta\left([\xi] x^{\prime} v\right)$ is $(C, G)$-irreducible. Suppose that $[h] u^{\prime \prime}$ is $(C \backslash\{c\})$-reducible and there is $c^{\prime} \in C \backslash\{c\}$ such that $\operatorname{lt}\left(c^{\prime}\right)=[h] u_{1}^{\prime \prime}$ with a prefix $u_{1}^{\prime \prime}$ of $u^{\prime \prime}$. Thus, $x=x^{\prime \prime} u_{2}^{\prime \prime}$ and $u_{2}^{\prime \prime} u_{1}^{\prime \prime} \in \operatorname{Dom}(G)$ for some $x^{\prime \prime}, u_{2}^{\prime \prime} \in \Sigma^{*}$. Because $x u^{\prime \prime}$ has no subword from $\operatorname{Dom}(G)$ other than $u$, we have $u_{2}^{\prime \prime} u_{1}^{\prime \prime}=u$, but this implies $c=c^{\prime}$, a contradiction. Therefore, $\operatorname{lt}(c)=[h] u^{\prime \prime}$ is $(C \backslash\{c\})$-irreducible, and we find that $C$ is normalized.

To see the confluence of $\rightarrow_{C}$ we need to consider critical pairs. Consider the element $c=[h] u^{\prime \prime}+\beta\left(t u^{\prime \prime}\right)-\beta\left([\xi] x^{\prime} v\right)$ as above, and suppose $u^{\prime \prime}=u_{1} u_{2}, u_{2} u_{3}-v^{\prime} \in$ $G$ for some $u_{1}, u_{2}, u_{3} \in \Sigma^{*}$, then we have a critical pair $\left(f_{1}, f_{2}\right)=\left(\beta\left([\xi] x^{\prime} v-t u^{\prime \prime}\right) \cdot u_{3}\right.$, $[h] u_{1} v^{\prime}$ ) for $C$. By Lemma 5.3 (and using Lemma 4.5) we have

$$
\begin{aligned}
f_{1} & =\beta\left([\xi] x^{\prime} v\right) \cdot u_{3}-\beta\left(t u^{\prime \prime}\right) \cdot u_{3} \\
& \Rightarrow^{*} \beta\left([\xi] x^{\prime} v u_{3}\right)-\beta\left(\left([\xi] x^{\prime} v\right) u_{3}\right)-\beta\left(t u^{\prime \prime} u_{3}\right)+\beta\left(\left(t u^{\prime \prime}\right) u_{3}\right) \\
& =\beta\left([\xi] x^{\prime} v u_{3}\right)-\beta\left(t u^{\prime \prime} u_{3}\right)=\beta\left([\xi] x u_{1} v^{\prime}\right)-\beta\left(t u_{1} v^{\prime}\right) \\
& =\beta([\xi] x z)-\beta(t z),
\end{aligned}
$$

where $z=\left(u_{1} v^{\prime} \hat{)}\right.$.

If $x z$ is $G$-irreducible, we have

$$
\beta([\xi] x z)=[h] z+\beta(t z) .
$$

Hence, we see $f_{1} \Rightarrow^{*}[h] z$ and $f_{2} \rightarrow_{G}^{*}[h] z$, that is, $f_{1} \downarrow_{C, G} f_{2}$.

If $x z$ is $G$-reducible, then $x=x_{1} x_{2}, z=z_{1} z_{2}$ and $x_{2} z_{1}-v^{\prime \prime} \in G$, where $x z_{1}$ is a minimal $G$-reducible word. Hence, we have the rule

$$
[h] z_{1} \rightarrow \beta\left([\xi] x_{1} v^{\prime \prime}\right)-\beta\left(t z_{1}\right)
$$

in $T_{C}$. Thus, again by Lemma 5.3 we have

$$
\begin{aligned}
f_{2} & \rightarrow_{G}^{*}[h] z_{1} z_{2} \\
& \Rightarrow_{C} \beta\left([\xi] x_{1} v^{\prime \prime}\right) z_{2}-\beta\left(t z_{1}\right) z_{2} \\
& \Rightarrow^{*} \beta\left([\xi] x_{1} v^{\prime \prime} z_{2}\right)-\beta\left(\left([\xi] x_{1} v^{\prime \prime}\right) z_{2}\right)-\beta\left(t z_{1} z_{2}\right)+\beta\left(\left(t z_{1}\right) z_{2}\right) \\
& =\beta([\xi] x z)-\beta(t z),
\end{aligned}
$$

because $\beta([\xi] x z)=\beta\left([\xi] x_{1} v^{\prime \prime} z_{2}\right)$ and $\left([\xi] x_{1} v^{\prime \prime}\right)=\left(t z_{1}\right)$. The last element in (14) is equal to the last element in (13), and we find $f_{1} \downarrow_{C, G} f_{2}$. Because any critical pair for $C$ is resolved, $\rightarrow_{C}$ is complete modulo $G$ by Proposition 4.3 .

\section{EXACT SEQUENCES OF BIMODUles}

Let $H$ be a very monic normalized Gröbner base of an $F$-subbimodule $L$ of $F \cdot X \cdot F$ modulo $G$. Then, $L$ is the $F$-subbimodule generated by $H$ and $I_{X}=$ $F \cdot X \cdot I+I \cdot X \cdot F$. We consider the free $F$-bimodule $F \cdot H \cdot F$ generated by the set $H$ and define the morphism $\delta: F \cdot H \cdot F \rightarrow F \cdot X \cdot F$ of $F$-bimodules by $\delta([h])=h$ for $h \in H$ as in the last section. Also we have a morphism $\partial: A \cdot H \cdot A \rightarrow A \cdot X \cdot A$ of $A$-bimodules defined by $\partial([h])=\rho_{X}(h)$ for $h \in H$.

Let $C$ be the Gröbner base made from proper critical pairs of $H$. We consider the free $F$-bimodule $F \cdot C \cdot F$ and the free $A$-bimodule $A \cdot C \cdot A$ generated by $C$. With $[c]$ denoting the generator corresponding to $c \in C$, we have a morphism $\delta^{\prime}: F \cdot C \cdot F \rightarrow F \cdot H \cdot F$ of $F$-bimodules and a morphism $\partial^{\prime}: A \cdot C \cdot A \rightarrow A \cdot H \cdot A$ 
of $A$-bimodules defined by $\delta^{\prime}([c])=c$, and $\partial^{\prime}([c])=\rho_{H}(c)$. With these morphisms we have a commutative diagram

$$
\begin{aligned}
& F \cdot C \cdot F \quad \stackrel{\delta^{\prime}}{\rightarrow} \quad F \cdot H \cdot F \quad \stackrel{\delta}{\rightarrow} \quad F \cdot X \cdot F \\
& \downarrow \rho_{C} \quad \downarrow \rho_{H} \quad \downarrow \rho_{X} \\
& A \cdot C \cdot A \stackrel{\partial^{\prime}}{\rightarrow} A \cdot H \cdot A \stackrel{\partial}{\rightarrow} A \cdot X \cdot A .
\end{aligned}
$$

We shall prove our second key result that the lower sequence in (15) is exact. To this end we need the following lemma involving the $K$-linear mapping $\beta: F \cdot X \cdot F \rightarrow$ $F \cdot H \cdot F$ defined in the last section.

Lemma 6.1. For $f \in F \cdot X \cdot F$ we have

$$
\delta \circ \beta(f) \equiv f-\tilde{f}\left(\bmod I_{X}\right) .
$$

Proof. By $K$-linearity of the mappings $\delta, \beta$ and ${ }^{\sim}$, we may suppose that $f=x[\xi] y$ with $x, y \in \Sigma^{*}$ and $\xi \in X$. Since $\beta(f)=\beta(\hat{f})$ and $f-\hat{f} \equiv 0\left(\bmod I_{X}\right)$, we may further suppose that $x, y \in \operatorname{Irr}(G)$. We prove the assertion by induction on $f$ with respect to $\succ$.

If $f$ is $H$-irreducible, then $\beta(f)=0$ and $f=\tilde{f}$, and both sides of (16) are 0. On the other hand, if $f$ is $H$-reducible, then $y=y^{\prime} y^{\prime \prime}, h=[\xi] y^{\prime}-t \in H$. Since

$$
\beta(f)=x[h] y^{\prime \prime}+\beta\left(x t y^{\prime \prime}\right)
$$

by the definition of $\beta$, we have

$$
\delta \circ \beta(f)=x\left([\xi] y^{\prime}-t\right) y^{\prime \prime}+\delta \circ \beta\left(x t y^{\prime \prime}\right) .
$$

Here, by the induction hypothesis we see

$$
\delta \circ \beta\left(x t y^{\prime \prime}\right) \equiv x t y^{\prime \prime}-\left(x t y^{\prime \prime}\right)\left(\bmod I_{X}\right) .
$$

Because $f=x[\xi] y^{\prime} y^{\prime \prime}$ and $\tilde{f}=\left(x t y^{\prime \prime}\right)$, we obtain the desired congruence in (16).

Lemma 6.2. $\rho_{X} \circ \delta \circ \delta^{\prime}=0$.

Proof. Let $h=[\xi] x-t \in H, x=x^{\prime} u^{\prime}, u=u^{\prime} u^{\prime \prime}, u-v \in G$ and $x^{\prime} u$ is a minimal $G$-reducible word. Then we have an element

$$
c=[h] u^{\prime \prime}+\beta\left(t u^{\prime \prime}\right)-\beta\left([\xi] x u^{\prime \prime}\right)
$$

of $C$. By Lemma 6.1 we obtain

$$
\begin{aligned}
\delta \circ \delta^{\prime}([c]) & =h \cdot u^{\prime \prime}+\delta \circ \beta\left(t u^{\prime \prime}\right)-\delta \circ \beta\left([\xi] x u^{\prime \prime}\right) \\
& \equiv([\xi] x-t) u^{\prime \prime}+t u^{\prime \prime}-\left(t u^{\prime \prime}\right)-[\xi] x u^{\prime \prime}+\left([\xi] x u^{\prime \prime}\right)\left(\bmod I_{X}\right) \\
& =-\left(t u^{\prime \prime}\right)+\left([\xi] x u^{\prime \prime}\right)=0 .
\end{aligned}
$$

It follows that $\rho_{X} \circ \delta \circ \delta^{\prime}([c])=0$, as desired.

Lemma 6.3. We have $\operatorname{Im}\left(\delta^{\prime}\right)+I_{H} \supset \operatorname{Ker}\left(\rho_{X} \circ \delta\right)$.

Proof. Let $f \in F \cdot H \cdot F$ such that $\rho_{X} \circ \delta(f)=0$. We shall show that $f \in \operatorname{Im}\left(\delta^{\prime}\right)+I_{H}$ by induction on $f$ with respect to $\succ$. Let $f=\sum k_{i} \cdot x_{i}\left[h_{i}\right] y_{i}$ with $k_{i} \in K \backslash\{0\}, x_{i}$, $y_{i} \in \Sigma^{*}$ and $h_{i}=\left[\xi_{i}\right] z_{i}-t_{i} \in H$, where $\xi_{i} \in X, z_{i} \in \operatorname{Irr}(G), t_{i} \in F \cdot H \cdot F$ and 
$x_{i}\left[h_{i}\right] y_{i}$ are different. Since $f-\hat{f} \in I_{H}$, we may suppose that $f$ is $G$-irreducible, that is, $x_{i}, y_{i} \in \operatorname{Irr}(G)$ for all $i$. Suppose $k_{1} \cdot x_{1}\left[h_{1}\right] y_{1}$ is the leading term of $f$. Since

$$
\begin{aligned}
\rho_{X} \circ \delta(f) & \left.=\rho_{X}\left(\sum k_{i} \cdot x_{i}\left(\left[\xi_{i}\right] z_{i}-t_{i}\right) y_{i}\right)\right) \\
& =\sum k_{i} \cdot \rho\left(x_{i}\right)\left[\xi_{i}\right] \rho\left(z_{i} y_{i}\right)-\sum k_{i} \cdot \rho_{X}\left(x_{i} t_{i} y_{i}\right) \\
& =0,
\end{aligned}
$$

the equality

$$
\sum k_{i} \cdot x_{i}\left[\xi_{i}\right]\left(z_{i} y_{i} \hat{)}-\sum k_{i} \cdot\left(x_{i} t_{i} y_{i} \hat{)}=0\right.\right.
$$

holds in $F \cdot X \cdot F$ by Proposition 3.4, (1). This implies that the word $z_{1} y_{1}$ is $G$ reducible. In fact, if $z_{1} y_{1}$ were $G$-irreducible, then $k_{1} x_{1}\left[\xi_{1}\right]\left(z_{1} y_{1} \hat{)}=k_{1} x_{1}\left[\xi_{1}\right] z_{1} y_{1}\right.$ would be greater than any other term in the left-hand side of (17) and would never be cancelled to 0 . Therefore, $z_{1}=z_{1}^{\prime} z_{1}^{\prime \prime}, y_{1}=y_{1}^{\prime} y_{1}^{\prime \prime}, z_{1}^{\prime \prime} y_{1}^{\prime}-v \in G$ and $z_{1} y_{1}^{\prime}$ has no proper $G$-reducible prefix. Thus, we have the element

$$
c=\left[h_{1}\right] y_{1}^{\prime}+\beta\left(t_{1} y_{1}^{\prime}\right)-\beta\left(\left[\xi_{1}\right] z_{1}^{\prime} v\right)
$$

in $C$. Set $f^{\prime}=f-\delta^{\prime}\left(k_{1} x_{1}[c] y_{1}^{\prime \prime}\right)$, then $f^{\prime} \in \operatorname{Ker}\left(\rho_{X} \circ \delta\right)$ by Lemma 6.2 , and

$$
f^{\prime}=f-k_{1} x_{1}\left[h_{1}\right] y_{1}-k_{1} x_{1} \beta\left(t_{1} y_{1}^{\prime}\right) y_{1}^{\prime \prime}-k_{1} x_{1} \beta\left(\left[\xi_{1}\right] z_{1}^{\prime} v\right) y_{1}^{\prime \prime} \prec f
$$

because the leading term $k_{1} x_{1}\left[h_{1}\right] y_{1}$ of $f$ is cancelled in $f^{\prime}$. By the induction hypothesis, $f^{\prime} \in \operatorname{Im}\left(\delta^{\prime}\right)+I_{H}$. It follows that $f=f^{\prime}+\delta^{\prime}\left(k_{1} x_{1}[c] y_{1}^{\prime \prime}\right)$ is also in $\operatorname{Im}\left(\delta^{\prime}\right)+I_{H}$.

By Lemmas 6.2 and 6.3 we see

$$
\operatorname{Im}\left(\delta^{\prime}\right)+I_{H}=\operatorname{Ker}\left(\rho_{X} \circ \delta\right)=\operatorname{Ker}(\delta)+I_{H} .
$$

Thus, $\delta^{\prime}([C])=C$ is a Gröbner base of $\operatorname{Ker}(\delta)+I_{H}$ by Theorem 5.4, and we obtain the main theorems in this section.

Theorem 6.4. $C$ is a normalized very monic Gröbner base of $\operatorname{Ker}(\delta)+I_{H}$ on $F \cdot H \cdot F$ modulo $G$, that is, $C$ is a Gröbner base for $\operatorname{Ker}(\partial)$.

Theorem 6.5. Let $M$ be defined by a normalized very monic Gröbner base $H$ on $F \cdot X \cdot F$ modulo $G$. We have an exact sequence of the free A-bimodules

$$
A \cdot C \cdot A \stackrel{\partial^{\prime}}{\rightarrow} A \cdot H \cdot A \stackrel{\partial}{\rightarrow} A \cdot X \cdot A \stackrel{\bar{\eta}}{\rightarrow} M \rightarrow 0,
$$

where $\bar{\eta}$ is the surjection in (4).

Proof. The morphism $\bar{\eta}$ is surjective, and $\operatorname{Ker}(\bar{\eta})=L_{A}(H)$, which is generated by $\rho_{X}(H)$ by Proposition 3.2, is equal to $\operatorname{Im}(\partial)$. Hence, the sequence is exact at $M$ and $A \cdot X \cdot A$. The equality (18) implies the sequence is also exact at $A \cdot H \cdot A$.

\section{Construction of free Bimodule Resolutions}

Let $M$ be an $A$-bimodule admitting a very monic Gröbner base, that is, $M \cong$ $M\left(X_{1}, G\right)$, where $X_{1}$ is a very monic Gröbner base modulo $G$ on the free $F$-bimodule $F \cdot X_{0} \cdot F$ generated by a set $X_{0}$ of generators of $M$. Due to Proposition 4.1, we may suppose that $X_{1}$ is normalized.

Let $X_{2}$ be the Gröbner base on $F \cdot X_{1} \cdot F$ made from proper critical pairs of $X_{1}$. By Theorem 6.5, we have an exact sequence of the free $A$-bimodules

$$
A \cdot X_{2} \cdot A \stackrel{\partial_{2}}{\rightarrow} A \cdot X_{1} \cdot A \stackrel{\partial_{1}}{\rightarrow} A \cdot X_{0} \cdot A \rightarrow M \rightarrow 0 .
$$


By Theorem $6.4, X_{2}$ is a Gröbner base for $\operatorname{Ker}\left(\partial_{1}\right)$. So, again by Theorem 6.5 , with the Gröbner base $X_{3}$ made from proper critical pairs of $X_{2}$, we have an exact sequence

$$
A \cdot X_{3} \cdot A \stackrel{\partial_{3}}{\rightarrow} A \cdot X_{2} \cdot A \stackrel{\partial_{2}}{\rightarrow} A \cdot X_{1} \cdot A \stackrel{\partial_{1}}{\rightarrow} \operatorname{Im}\left(\partial_{1}\right) .
$$

Combining this with (19) we have an exact sequence

$$
A \cdot X_{3} \cdot A \stackrel{\partial_{3}}{\rightarrow} A \cdot X_{2} \cdot A \stackrel{\partial_{2}}{\rightarrow} A \cdot X_{1} \cdot A \stackrel{\partial_{1}}{\rightarrow} A \cdot X_{0} \cdot A \rightarrow M \rightarrow 0 .
$$

We can repeat this construction arbitrarily many times and we have a free $A$ bimodule resolution of $M$

$$
\mathbf{X}: \cdots \stackrel{\partial_{n+1}}{\rightarrow} A \cdot X_{n} \cdot A \stackrel{\partial_{n}}{\rightarrow} \cdots \stackrel{\partial_{1}}{\rightarrow} A \cdot X_{0} \cdot A \rightarrow M \rightarrow 0 .
$$

If $G$ and $X_{0}$ is finite, $X_{n}$ are finite for all $n$, and hence all the free $A$-bimodules in (20) are finitely generated. Summarizing:

Theorem 7.1. If an algebra $A$ over $K$ admits a Gröbner base $G$ and an $A$-bimodule $M$ admits a Gröbner base $X_{1}$ modulo $G$ on a set $X_{0}$ of generators of $M$, then we have a free bimodule resolution $\mathbf{X}$ in (20) of $M$. If $G, X_{0}$ and $X_{1}$ are finite, all the free bimodules in $\mathbf{X}$ are of finite rank.

We say an $A$-bimodule $M$ has type $\mathrm{FP}_{n}$ if it has a partial $A$-bimodule resolution

$$
F_{n} \stackrel{\partial_{n}}{\rightarrow} \cdots \stackrel{\partial_{1}}{\rightarrow} F_{0} \rightarrow M \rightarrow 0
$$

such that $F_{i}(0 \leq i \leq n)$ are free of finite rank. $M$ has type $\mathrm{FP}_{\infty}$ if it has type $\mathrm{FP}_{n}$ for all $n \geq 0$.

Corollary 7.2. If an algebra $A$ over $K$ admits a finite Gröbner base $G$, then a finitely generated A-bimodule with finite Gröbner base modulo $G$ has type $\mathrm{FP}_{\infty}$.

Now we perform this construction for the $A$-bimodule $A$. Without loss of generality, we may assume that $\operatorname{Dom}(G) \cap \Sigma=\emptyset$. First, we define the order $\succ$ on the product $\Sigma^{*} \times \Sigma^{*}$ as follows. For $x, y, x^{\prime}, y^{\prime} \in \Sigma^{*},(x, y) \succ\left(x^{\prime}, y^{\prime}\right)$ holds, if $x y \succ x^{\prime} y^{\prime}$ in $\Sigma^{*}$, or $x y=x^{\prime} y^{\prime}$ and $|y|>\left|y^{\prime}\right|$. Clearly, this is a well-order and can be extended to a well-founded partial order $\succ$ on the free $F$-bimodule $F \cdot[] \cdot F$ with a single generator [ ], which is a $K$-space with base $\Sigma^{*} \times \Sigma^{*}$.

Let

$$
X_{1}=\{[] a-a[] \mid a \in \Sigma\} \subset F \cdot[] \cdot F .
$$

We have the augmentation mapping $\epsilon$ from the cyclic free $A$-bimodule $A \cdot[] \cdot A$ to $A$,

and the surjection

$$
\epsilon: A \cdot[] \cdot A \rightarrow A, \epsilon(x[] y)=x y,
$$

$$
\rho_{[]}: F \cdot[] \cdot F \rightarrow A \cdot[] \cdot A .
$$

Lemma 7.3. $X_{1}$ is a normalized very monic Gröbner base on $F \cdot[] \cdot F$ for $\operatorname{Ker}(\epsilon)$ modulo $G$.

Proof. Since [ ] $\succ \succ a[]$, [ ] $a$ is the leading term of [ ] $a-a\left[\right.$ ], and so $X_{1}$ is normalized and very monic. Next we show the confluence. Let $u-v \in G$ and let $a_{1}$ be the first letter of $u$, then we have a proper critical pair ([ ] $\left.a_{1} u^{\dagger} \rightarrow a_{1}[] u^{\dagger},[] u \rightarrow[] v\right)$ of reductions, where $u^{\dagger}$ denotes the maximal proper suffix of $u ; u=a_{1} u^{\dagger}$. Since

$$
a_{1}[] u^{\dagger} \rightarrow_{X_{1}}^{*} u[] \rightarrow_{G} v[]
$$


and

$$
[] v \rightarrow{ }_{X_{1}}^{*} v[]
$$

the critical pair is resolvable. Hence, $X_{1}$ is a Gröbner base, and clearly it generates $\operatorname{Ker}(\epsilon)$.

Let $F \cdot \Sigma \cdot F$ (resp. $A \cdot \Sigma \cdot A$ ) be the free $F$-bimodule (resp. $A$-bimodule) generated by $\Sigma$, and let $[a]_{1}$ denote the generator of it corresponding to $a \in \Sigma$. In general, for $x=a_{1} \cdots a_{n} \in \Sigma^{*},[x]_{1}$ denotes the element

$$
[x]_{1}=\left[a_{1}\right]_{1} a_{2} \cdots a_{n}+a_{1}\left[a_{2}\right]_{1} a_{3} \cdots a_{n}+\cdots+a_{1} \cdots a_{n-1}\left[a_{n}\right]_{1}
$$

of $F \cdot \Sigma \cdot F$. This $[x]_{1}$ is also considered to be an element of $A \cdot \Sigma \cdot A$ modulo $I_{\Sigma}$. We extend the mapping [ $]_{1}$ to the mapping [ $]_{1}: F \rightarrow F \cdot \Sigma \cdot F, K$-linearly. Let $\delta_{1}: F \cdot \Sigma \cdot F \rightarrow F \cdot[] \cdot F$ (resp. $\partial_{1}: A \cdot \Sigma \cdot A \rightarrow A \cdot[] \cdot A$ ) be the morphism of $F$-bimodules (resp. $A$-bimodules) defined by

$$
\delta_{1}\left([a]_{1}\right)=[] a-a[]\left(\operatorname{resp} . \partial_{1}\left([a]_{1}\right)=[] a-a[]\left(\bmod I_{[]}\right)\right) .
$$

For the proper critical pair $\left([] a_{1} u^{\dagger} \rightarrow a_{1}[] u^{\dagger},[] u \rightarrow[] v\right)$ of reductions for $u-v \in G$ with $u=a_{1} u^{\dagger}$ in the proof of Lemma 7.3 above, we associate an element

$$
g=\left[a_{1}\right]_{1} u^{\dagger}+\beta\left(a_{1}[] u^{\dagger}\right)-\beta([] v),
$$

in $F \cdot[] \cdot F$, where $\beta: F \cdot[] \cdot F \rightarrow F \cdot \Sigma \cdot F$ is the morphism of $K$-modules defined in Section 5. By definition we have

$$
\beta\left(a_{1}[] u^{\dagger}\right)=a_{1}\left[a_{2}\right]_{1} a_{3} \cdots a_{m}+\cdots+a_{1} a_{2} \cdots a_{m-1}\left[a_{m}\right]_{1}=a_{1}\left[u^{\dagger}\right]_{1},
$$

and

$$
\beta([] v)=[v]_{1},
$$

where $u=a_{1} a_{2} \cdots a_{m}$. Hence, we see

$$
g=\left[a_{1}\right]_{1} u^{\dagger}+a_{1}\left[u^{\dagger}\right]_{1}+[v]_{1}=[u]_{1}-[v]_{1} .
$$

Thus,

Lemma 7.4. $X_{2}=\left\{[u]_{1}-[v]_{1} \mid u-v \in G\right\}$ forms a normalized very monic Gröbner base on $F \cdot \Sigma \cdot F$ for Ker $\left(\partial_{1}\right)$.

Define a morphism $\partial_{2}: A \cdot G \cdot A \rightarrow A \cdot \Sigma \cdot A$ by

$$
\partial_{2}\left([g]_{2}\right)=[u]_{1}-[v]_{1}
$$

for $g=u-v \in G$, where $[g]_{2}$ denotes the formal generator of the free $A$-bimodule $A \cdot G \cdot A$ corresponding to $g \in G$.

Next, we consider a pair $c=\left(g_{1}, g_{2}\right)$ of rules in $G$ such that $g_{1}=u_{1}-v_{1}$, $g_{2}=u_{2}-v_{2} \in G, u_{1}=u_{1}^{\prime} z, u_{2}=z u_{2}^{\prime}, z \neq 1, u_{1}^{\prime}=a u_{1}^{\dagger}$ and $u_{1}^{\dagger} u_{2}^{\prime}$ is a minimal $G$-reducible word. We call such a pair $\left(g_{1}, g_{2}\right)$ a proper critical pair of reductions in $G$. Then, we have a rule

$$
\left[g_{1}\right]_{2}:[a]_{1} u_{1}^{\dagger} z \rightarrow-a\left[u_{1}^{\dagger} z\right]_{1}+\left[v_{1}\right]_{1}
$$

in $X_{2}$ and a proper critical pair

$$
\left([a]_{1} u_{1}^{\dagger} u_{2} \rightarrow-a\left[u_{1}^{\dagger} z\right]_{1} u_{2}^{\prime}+\left[v_{1}\right]_{1} u_{2}^{\prime},[a]_{1} u_{1}^{\dagger} u_{2} \rightarrow[a]_{1} u_{1}^{\dagger} v_{2}\right)
$$

of reductions for $X_{2}$. So we obtain a rule

$$
\begin{gathered}
{\left[g_{1}\right]_{2} \cdot u_{2}^{\prime} \rightarrow \beta\left([a]_{1} u_{1}^{\dagger} v_{2}\right)+\beta\left(a\left[u_{1}^{\dagger} z\right] u_{2}^{\prime}-\beta\left(\left[v_{1}\right]_{1} u_{2}^{\prime}\right)\right.} \\
=\beta\left(\left[u_{1}^{\prime}\right]_{1} v_{2}\right)+\beta\left(u_{1}^{\prime}[z] u_{2}^{\prime}\right)-\beta\left(\left[v_{1}\right]_{1} u_{2}^{\prime}\right) .
\end{gathered}
$$


on $F \cdot G \cdot F$. Here, since $u_{1}^{\prime}[z] u_{2}^{\prime}$ is $\left(X_{2}, G\right)$-irreducible, $\beta\left(u_{1}^{\prime}[z] u_{2}^{\prime}\right)=0$. Thus we have a rule

$$
\left[g_{1}\right]_{2} \cdot u_{2}^{\prime} \rightarrow \beta\left(\left[u_{1}^{\prime}\right]_{1} v_{2}\right)-\beta\left(\left[v_{1}\right]_{1} u_{2}^{\prime}\right) .
$$

Let $C$ be the set of all proper critical pairs of rules in $G$, and for $c=\left(g_{1}, g_{2}\right) \in C$ as above we associate the element

$$
c_{2}=\left[g_{1}\right]_{2} \cdot u_{2}^{\prime}+\beta\left(\left[v_{1}\right]_{1} u_{2}^{\prime}\right)-\beta\left(\left[u_{1}^{\prime}\right]_{1} v_{2}\right)
$$

of $F \cdot G \cdot F$.

Lemma 7.5. The set $X_{3}$ of the elements (21) corresponding to all elements $c$ in $C$ forms a normalized very monic Gröbner base on $F \cdot G \cdot F$ for $\operatorname{Ker}\left(\partial_{2}\right)$.

Defining a morphism $\partial_{3}: A \cdot C \cdot A \rightarrow A \cdot G \cdot A$ by $\partial_{3}\left([c]_{3}\right)=\rho_{G}\left(c_{2}\right)$ for $c \in C$, where $[c]_{3}$ denotes the formal generator of $A \cdot C \cdot A$ corresponding to $c$ and $c_{2}$ is the element given in (21), we have an exact sequence

$$
A \cdot C \cdot A \stackrel{\partial_{3}}{\rightarrow} A \cdot G \cdot A \stackrel{\partial_{2}}{\rightarrow} A \cdot \Sigma \cdot A \stackrel{\partial_{1}}{\rightarrow} A \cdot[] \cdot A \stackrel{\epsilon}{\rightarrow} A \rightarrow 0
$$

We can continue this construction further, but calculations become more and more complex, so we stop here. Though it is difficult to give explicit forms of elements of our Gröbner base in higher dimension, we can give the leading terms of elements of the Gröbner base in a systematic way.

We define a directed graph $\Gamma$ associated with $G$ as follows. The set $V$ of vertices in $\Gamma$ is the union of $\Sigma$ and the set of nonempty proper suffixes of words in $\operatorname{Dom}(G)$. For $x, y \in V$, there is an edge from $x$ to $y$, if and only if $x y$ is a minimal $G$-reducible word, that is, $x=x^{\prime} x^{\prime \prime}, x^{\prime \prime} y \in G$ and $x y$ has no proper $G$-reducible subword. $\Gamma$ is a finite graph if $G$ is finite, but it is infinite in general. For $n>0, C_{n}$ denotes the set of all directed paths in $\Gamma$ of length $n-1$ starting with some $a \in \Sigma$.

In particular, $C_{1}$ is the set of trivial paths $\iota_{a}$ at $a$ for all $a \in \Sigma$. Thus, it is bijective to $\Sigma$. $C_{2}$ is the set of all edges $a \rightarrow u^{\prime}\left(a \in \Sigma, a u^{\prime} \in \operatorname{Dom}(G)\right)$ in $\Gamma$. It is bijective to $G$. $C_{3}$ is the set of all paths $a \rightarrow u^{\prime} \rightarrow u^{\prime \prime}$ in $\Gamma$ and it is bijective to the set of all proper critical pairs of reductions in $G$.

Theorem 7.6. We have a free A-bimodule resolution of $A$ :

$$
\mathbf{H}: \rightarrow A \cdot C_{n} \cdot A \stackrel{\partial_{n}}{\rightarrow} A \cdot C_{n-1} \cdot A \rightarrow \cdots \rightarrow A \cdot C_{1} \cdot A \stackrel{\partial_{1}}{\rightarrow} A \cdot[] \cdot A \stackrel{\epsilon}{\rightarrow} A .
$$

Here, $\operatorname{Ker}\left(\partial_{n-1}\right)$ has a very monic normalized Gröbner base $\left\{h_{c} \mid c \in C_{n}\right\}$ on $F$. $C_{n-1} \cdot F$ such that $\operatorname{lt}\left(h_{c}\right)=\left[c^{\prime}\right] v_{n-1}$ and $\partial_{n}([c])=\rho_{C_{n-1}}\left(h_{c}\right)$, where $c$ is a path $a \rightarrow v_{1} \rightarrow \cdots \rightarrow v_{n-2} \rightarrow v_{n-1}$ of length $n-1$ in $\Gamma$ and $c^{\prime}$ is the subpath $a \rightarrow v_{1} \rightarrow$ $\cdots \rightarrow v_{n-2}$ of $c$ of length $n-2$.

Proof. By induction on $n$. Assume that the resolution is constructed up to $n$ and $\left\{h_{c} \mid c \in C_{n}\right\}$ forms a Gröbner base for $\operatorname{Ker}\left(\partial_{n-1}\right)$, where $h_{c}=\left[c^{\prime}\right] v_{n-1}-t$ with $t \in$ $F \cdot C_{n-1} \cdot F$. Suppose that $c$ is prolonged to a path $\bar{c}=a \rightarrow v_{1} \rightarrow \cdots \rightarrow v_{n-1} \rightarrow v_{n}$ in $\Gamma$ of length $n$, that is, there is $v_{n} \in \Sigma^{*}$ such that $v_{n-1} v_{n}$ is a minimal $G$-reducible word. Then we have a proper critical pair $\left(\left[c^{\prime}\right] v_{n-1} v_{n} \rightarrow t v_{n},\left[c^{\prime}\right] v_{n-1} v_{n} \rightarrow_{G}\left[c^{\prime}\right] v^{\prime}\right)$ of reductions. The collection of these elements

$$
h_{\bar{c}}=[c] v_{n}+\beta\left(t \cdot v_{n}\right)-\beta\left(\left[c^{\prime}\right] v^{\prime}\right)
$$

of $F \cdot C_{n} \cdot F$ for $\bar{c} \in C_{n+1}$ forms a Gröbner base for $\operatorname{Ker}\left(\partial_{n}\right)$, and the resolution is prolonged up to $n+1$ with $\partial_{n+1}: A \cdot C_{n+1} \cdot A \rightarrow A \cdot C_{n} \cdot A$ defined by $\partial_{n+1}([\bar{c}])=$ $\rho_{X_{n}}\left(h_{\bar{c}}\right)$ in virtue of Theorems 6.4 and 6.5 . 
For two $A$-bimodules $M$ and $N$ let $\operatorname{Hom}_{A, A}(M, N)$ be the $K$-modules consisting of all bimodule morphisms from $M$ to $N$. Taking the functor $\operatorname{Hom}_{A, A}(., A)$ on (22), we have a complex

$$
\operatorname{Hom}_{A, A}(\mathbf{H}, A): 0 \rightarrow A \stackrel{\partial_{1}^{*}}{\rightarrow} A^{d_{1}} \rightarrow \cdots \rightarrow A^{d_{n-1}} \stackrel{\partial_{n}^{*}}{\rightarrow} A^{d_{n}} \stackrel{\partial_{n+1}^{*}}{\rightarrow} A^{d_{n+1}} \rightarrow \cdots,
$$

where $d_{i}=\left|C_{i}\right|$. Since $A$ is free as $K$-module by Corollary 2.5, the homology group $H^{n}(A)=\operatorname{Ker}\left(\partial_{n+1}^{*}\right) / \operatorname{Im}\left(\partial_{n}^{*}\right)$ is equal to the Hochschild cohomology of $A$ of dimension $n$.

Because $A$ is a free $K$-module we can compute other (co)homology groups with our complex $\mathbf{H}$ as below (see [7, Chaps. 9, 10). Because $A \cdot C_{n} \cdot A=A \otimes_{K} K C_{n} \otimes_{K} A$ is free as a left $A$-module, $\mathbf{H}$ is considered to be a free left $A$-module resolution of $A$. Hence, for any right $A$-module $M$, the complex $M \otimes_{A} \mathbf{H}$ is exact because $H_{n}\left(M \otimes_{A} \mathbf{H}\right)=\operatorname{Tor}_{n}^{A}(M, A)=0$ for any $n>0$. Moreover, if $M$ is projective as a $K$-module, then $\mathbf{H} \otimes_{A} M$ is a projective resolution of $M$. Hence, we have the following isomorphism of $K$-modules:

$$
\operatorname{Tor}_{n}^{A}(M, N) \cong H_{n}\left(M \otimes_{A} \mathbf{H} \otimes_{A} N\right)
$$

for any left $A$-module $N$. For the same reason the complex $\operatorname{Hom}_{A}(\mathbf{H}, M)$ is exact for any left $A$-module $M$, and if $M$ is $K$-projective, $\operatorname{Hom}_{A}(\mathbf{H}, M)$ is a projective resolution of $M$. Hence,

$$
\operatorname{Ext}_{A}^{n}(N, M) \cong H^{n}\left(\operatorname{Hom}_{A}\left(N, \operatorname{Hom}_{A}(\mathbf{H}, M)\right)\right.
$$

for any left $A$-module $N$. Furthermore, since $\operatorname{Hom}_{A}\left(N, \operatorname{Hom}_{A}(\mathbf{H}, M)\right)$ is naturally isomorphic to $\operatorname{Hom}_{A, A}\left(\mathbf{H}, \operatorname{Hom}_{K}(N, M)\right)$, we have

$$
\operatorname{Ext}_{A}^{n}(N, M) \cong H^{n}\left(\operatorname{Hom}_{A, A}\left(\mathbf{H}, \operatorname{Hom}_{K}(N, M)\right)\right) .
$$

If $A$ is a supplemented algebra over $K$ with an augmentation $\bar{\epsilon}: A \rightarrow K$, then $K$ itself is an $A$-bimodules via $\bar{\epsilon}$. Letting $N=M=K$ in the above isomorphisms we have

$$
\operatorname{Tor}_{n}^{A}(K, K) \cong H_{n}\left(K \otimes_{A} \mathbf{H} \otimes_{A} K\right)
$$

and

$$
\operatorname{Ext}_{A}^{n}(K, K) \cong H^{n}\left(\operatorname{Hom}_{A, A}(\mathbf{H}, K)\right) .
$$

\section{EXAMPLES}

In this section we compute our resolutions for some example algebras. Though we do not attempt a systematical application of our methods, we show how our construction works for a several typical types of algebras. Even if we get a resolution, it is not an easy task to calculate the Hochschild cohomology. So, we give a detailed calculation of the cohomology only in the first example.

Example 8.1. (1) Let $U$ be a subset of $\Sigma^{2}$ and $\phi: U \rightarrow K \cdot \Sigma \oplus K$ be a mapping. Let $\phi^{\prime}: K \cdot \Sigma^{2} \oplus K \cdot \Sigma \rightarrow K \cdot \Sigma^{2} \oplus K \cdot \Sigma \oplus K$ be the $K$-linear mapping defined by

$$
\phi^{\prime}(u)= \begin{cases}\phi(u) & \text { if } u \in U, \\ u & \text { if } u \notin U,\end{cases}
$$

for $u \in \Sigma^{\leq 2}$. Suppose that

$$
\phi^{\prime}(\phi(a b) c)=\phi^{\prime}(a \phi(b c))
$$


holds for any $a, b, c \in \Sigma$ such that $a b, b c \in U$. Let $I$ be the ideal of the free algebra $F=K \cdot \Sigma^{*}$ generated by $G=\{u-\phi(u)\}$, and let $A=F / I$. Then, $G$ is a Gröbner base of $I$. In fact, (223) guarantees the confluence of the rewriting system $\{u \rightarrow \phi(u) \mid u \in U\}$.

For $n \geq 0$, let $V_{n}$ be the set of words $v$ of length $n$ such that any subword of $v$ of length 2 belongs to $U$. For example, $V_{0}=\{1\}, V_{1}=\Sigma, V_{2}=U$ and $V_{3}=$ $\{a b c \mid a, b, c \in \Sigma, a b, b c \in U\}$. As before, $[v]_{n}$ for $v \in V_{n}$ denotes the formal generator corresponding to $v$, and we extend this notation for any $K$-linear combination $x=\sum k_{i} v_{i} \in K \cdot \Sigma^{n}$ as $[x]_{n}=\sum_{i} k_{i}\left[v_{i}\right]_{n}$. In particular, $[x]_{n}=0$ for $x \in \Sigma \leq n \backslash V_{n}$. Moreover, for $x_{1}, \ldots, x_{n} \in K \cdot \Sigma \oplus K,\left[x_{1} \cdots x_{n}\right]_{n}$ is defined by expanding the product $x_{1} \cdots x_{n}$ to an element of $K \cdot \Sigma \leq n$.

Now, define an augmentation mapping $\partial_{0}=\bar{\epsilon}: A \cdot V_{0} \cdot A \rightarrow A$ and a morphism $\partial_{n}: A \cdot V_{n} \cdot A \rightarrow A \cdot V_{n-1} \cdot A$ of $A$-bimodules by $\epsilon([])=1, \partial_{1}\left([a]_{1}\right)=[] a-a[]$ for $a \in \Sigma$, and for $n \geq 2$,

$$
\begin{aligned}
\partial_{n}\left(\left[a_{1} \cdots a_{n}\right]_{n}\right)= & {\left[a_{1} \cdots a_{n-1}\right]_{n-1} a_{n}+(-1)^{n} a_{1}\left[a_{2} \cdots a_{n}\right]_{n-1} } \\
& +\sum_{i=1}^{n-1}(-1)^{n-i}\left[a_{1} \cdots a_{i-1} \phi\left(a_{i} a_{i+1}\right) a_{i+2} \cdots a_{n}\right]_{n-1},
\end{aligned}
$$

where $a_{1} \cdots a_{n} \in V_{n}$.

We claim that $\left(A \cdot M_{n} \cdot A, \partial_{n}\right)$ is a free $A$-bimodule resolution of $A$. More precisely, we shall show that $H_{n}=\delta_{n}\left(\left[V_{n}\right]_{n}\right)$ forms a normalized very monic Gröbner base for $\operatorname{Ker}\left(\partial_{n-1}\right)$ on $F \cdot V_{n-1} \cdot F$ modulo $G$, where $\delta_{n}: F \cdot V_{n} \cdot F \rightarrow F \cdot V_{n-1} \cdot F$ is the $F$-bilinear mapping defined as $\partial_{n}$. Since $\operatorname{lt}\left(\delta_{n}\left(\left[a_{1} \cdots a_{n}\right]_{n}\right)\right)=\left[a_{1} \cdots a_{n-1}\right]_{n-1} a_{n}$, $H_{n}$ is very monic and normalized. We shall show that $H_{n}$ is a Gröbner base for $\operatorname{Ker}\left(\partial_{n-1}\right)$ by induction on $n$. First, the elements $\delta_{2}\left([a b]_{2}\right)=[a]_{1} b+a[b]_{1}-[\phi(a b)]_{1}$ for $a b \in U$ actually form a Gröbner base for $\operatorname{Ker}\left(\partial_{1}\right)$ as we already saw in Section 7 , and the assertion is true for $n \leq 2$.

Suppose that $n \geq 2$ and $H_{n}$ forms a Gröbner base for $\operatorname{Ker}\left(\partial_{n-1}\right)$. For $x=$ $a_{1} \cdots a_{n} \in V_{n}$ and $a_{n+1} \in \Sigma$ such that $a_{n} a_{n+1} \in U, \operatorname{lt}\left(\delta_{n}\left([x]_{n}\right)\right)=\left[a_{1} \cdots a_{n-1}\right]_{n-1} a_{n}$ overlaps with $a_{n} a_{n+1}$ and we have a proper critical pair of reductions

$$
\left[a_{1} \cdots a_{n-1}\right]_{n-1} a_{n} a_{n+1} \rightarrow_{G}\left[a_{1} \cdots a_{n-1}\right]_{n-1} \phi\left(a_{n} a_{n+1}\right)
$$

and

$$
\begin{aligned}
{\left[a_{1} \cdots a_{n-1}\right]_{n-1} a_{n} a_{n+1} \rightarrow_{H_{n}} } & -(-1)^{n} a_{1}\left[a_{2} \cdots a_{n}\right]_{n-1} a_{n+1} \\
& -\sum_{i=1}^{n-1}(-1)^{n-i}\left[a_{1} \cdots a_{i-1} \phi\left(a_{i} a_{i+1}\right) a_{i+2} \cdots a_{n}\right]_{n-1} a_{n+1} .
\end{aligned}
$$

Thus we have an element

$$
\begin{aligned}
{\left[a_{1} \cdots a_{n}\right]_{n} a_{n+1}-} & \beta\left(\left[a_{1} \cdots a_{n-1}\right]_{n-1} \phi\left(a_{n} a_{n+1}\right)\right)-(-1)^{n} \beta\left(a_{1}\left[a_{2} \cdots a_{n}\right]_{n-1} a_{n+1}\right) \\
& -\sum_{i=1}^{n-1}(-1)^{n-i} \beta\left(\left[a_{1} \cdots a_{i-1} \phi\left(a_{i} a_{i+1}\right) a_{i+2} \cdots a_{n}\right]_{n-1} a_{n+1}\right) \\
= & {\left[a_{1} \cdots a_{n}\right]_{n} a_{n+1}+(-1)^{n+1} a_{1}\left[a_{2} \cdots a_{n+1}\right]_{n} } \\
& +\sum_{i=1}^{n}(-1)^{n-i+1}\left[a_{1} \cdots a_{i-1} \phi\left(a_{i} a_{i+1}\right) a_{i+2} \cdots a_{n+1}\right]_{n}
\end{aligned}
$$


of $F \cdot V_{n} \cdot F$. The collection of these elements gives rise to the Gröbner base $H_{n+1}$ for $\operatorname{Ker}\left(\partial_{n}\right)$ modulo $G$ by Theorem 6.4.

(2) Here, we consider the extreme case where $U=\Sigma^{2}$. Then, $\Sigma \cup\{1\}$ is a $K$-base of $A, \phi_{\mid \Sigma^{2}}^{\prime}=\phi$ and (23) is just the associative law. Thus, $\phi: \Sigma \rightarrow K \cdot \Sigma \oplus K$ is nothing but a multiplication table for $A$. We have $V_{n}=\Sigma^{n}$ and the resolution $\cdots \rightarrow A \cdot \Sigma^{n} \cdot A \stackrel{\partial_{n}}{\rightarrow} A \cdot \Sigma^{n-1} \cdot A \rightarrow \cdots$ with the differentiations $\partial_{n}$ given by (24) is the (modified) standard complex of $A$ ([7], Chap. 9, Sect. 6). If $\Sigma$ is restricted to a finite alphabet, $A$ is finite dimensional over $K$. However, our construction, in fact, remains valid even if $\Sigma$ is infinite. If we are allowed to use an infinite alphabet, any algebra over $K$ can be expressed in this way with a multiplication table. In this sense formula (24) is general.

(3) Next, let us consider a very special example, where $\Sigma=\{a, b, c\}, U=$ $\left\{a^{2}, a b, a c\right\}$ and

$$
\phi\left(a^{2}\right)=1, \phi(a b)=b, \phi(a c)=-c .
$$

It is easy to see that $\phi$ satisfies (23), and so $G=\left\{a^{2}-1, a b-b, a c+c\right\}$ is a Gröbner base on $K \cdot \Sigma^{*}$. The algebra $A=K \cdot \Sigma^{*} / I(G)$ has a $K$-linear base

$$
\operatorname{Irr}(G)=\{b, c\}^{*} a \cup\{b, c\}^{*},
$$

and any element $x$ of $A$ is uniquely written as

$$
x=\sum k_{i} \cdot b x_{i} a+\sum k_{i}^{\prime} \cdot c x_{i} a+\sum \ell_{i} \cdot b x_{i}+\sum \ell_{i}^{\prime} \cdot c x_{i}+k \cdot a+\ell
$$

with $k_{i}, k_{i}^{\prime}, \ell_{i}, \ell_{i}^{\prime}, k, \ell \in K$ and $x_{i} \in\{b, c\}^{*}$, where only a finite number of $k_{i}, k_{i}^{\prime}, \ell_{i}, \ell_{i}^{\prime}$ are nonzero.

We have $V_{0}=\{1\}$, and $V_{n}=\left\{a^{n}, a^{n-1} b, a^{n-1} c\right\}$ for $n \geq 1$. The differentations $\partial_{n}: A \cdot V_{n} \cdot A \rightarrow A \cdot V_{n-1} \cdot A$ are given by specializing (24) as follows:

$$
\partial_{1}([a])=[] a-a[], \partial_{1}([b])=[] b-b[], \partial_{1}([c])=[] c-c[],
$$

and

$$
\begin{gathered}
\partial_{n}\left(\left[a^{n}\right]_{n}\right)=\left[a^{n-1}\right]_{n-1} a+(-1)^{n} a\left[a^{n-1}\right]_{n-1}, \\
\partial_{n}\left(\left[a^{n-1} b\right]_{n}\right)=\left[a^{n-1}\right]_{n-1} b+(-1)^{n} a\left[a^{n-2} b\right]_{n-1}-\left[a^{n-2} b\right], \\
\partial_{n}\left(\left[a^{n-1} c\right]_{n}\right)=\left[a^{n-1}\right]_{n-1} c+(-1)^{n} a\left[a^{n-2} c\right]_{n-1}+\left[a^{n-2} c\right]
\end{gathered}
$$

for $n \geq 2$. Since $\left|V_{n}\right|=3$ for $n \geq 1$, we can give our resolution simply as

$$
\cdots \stackrel{\partial}{\rightarrow} A \cdot \Sigma \cdot A \stackrel{\bar{\partial}}{\rightarrow} A \cdot \Sigma \cdot A \stackrel{\partial}{\rightarrow} \cdots \stackrel{\bar{\partial}}{\rightarrow} A \cdot \Sigma \cdot A \stackrel{\partial}{\rightarrow} A \cdot \Sigma \cdot A \stackrel{\partial_{1}}{\rightarrow} A[] A \stackrel{\epsilon}{\rightarrow} A,
$$

where $\partial$ and $\bar{\partial}$ are $A$-bimodule morphisms from $A \cdot \Sigma \cdot A$ to $A \cdot \Sigma \cdot A$ given by

$$
\partial([a])=[a] a+a[b], \partial([b])=[a] b+a[b]-[b], \partial([c])=[a] c+a[c]+[c]
$$

and

$$
\bar{\partial}([a])=[a] a-a[b], \bar{\partial}([b])=[a] b-a[b]-[b], \bar{\partial}([c])=[a] c-a[c]+[c] .
$$

Here, for simplicity, we assume that $K$ is a field. First, suppose that the characteristic of $K$ is not equal to 2, and we shall compute the cohomology $H^{n}(A)$. Taking the functor $\operatorname{Hom}_{A, A}(., A)$ on (26), we have a complex

$$
A \stackrel{\partial_{1}^{*}}{\rightarrow} A^{3} \stackrel{\partial^{*}}{\rightarrow} A^{3} \stackrel{\bar{\partial}^{*}}{\rightarrow} A^{3} \stackrel{\partial^{*}}{\rightarrow} A^{3} \stackrel{\bar{\partial}^{*}}{\rightarrow} \cdots,
$$


where $\partial_{1}^{*}, \partial^{*}$ and $\bar{\partial}^{*}$ are $K$-linear mappings given by

$$
\begin{gathered}
\partial_{1}^{*}(x)=(x a-a x, x b-b x, x c-c x), \\
\partial^{*}(x, y, z)=(x a+a x, x b+a y-y, x c+a z+z), \\
\bar{\partial}^{*}(x, y, z)=(x a-a x, x b-a y-y, x c-a z+z),
\end{gathered}
$$

for $x, y, z \in A$. Let $\theta, \bar{\theta}, \gamma, \bar{\gamma}, \eta_{b}$ and $\eta_{c}$ be $K$-linear mappings from $A$ to $A$ defined by

$$
\begin{gathered}
\theta(x)=x a+a x, \bar{\theta}(x)=x a-a x, \\
\gamma(x)=x+a x, \bar{\gamma}(x)=x-a x, \\
\eta_{b}(x)=x b, \eta_{c}(x)=x c
\end{gathered}
$$

for $x \in A$. Then,

$$
\partial^{*}(x, y, z)=\left(\theta(x),-\bar{\gamma}(y)+\eta_{b}(x), \gamma(z)+\eta_{c}(x)\right)
$$

and

$$
\bar{\partial}^{*}(x, y, z)=\left(\bar{\theta}(x),-\gamma(y)+\eta_{b}(x), \bar{\gamma}(z)+\eta_{c}(x)\right) .
$$

We claim that

$$
\begin{gathered}
\operatorname{Ker}(\theta)=\operatorname{Im}(\bar{\theta})=K \cdot b\{b, c\}^{*}(a-1) \oplus K \cdot c\{b, c\}^{*}(a+1), \\
\operatorname{Ker}(\bar{\theta})=\operatorname{Im}(\theta)=K \cdot b\{b, c\}^{*}(a+1) \oplus K \cdot c\{b, c\}^{*}(a-1) \oplus K \cdot\{a, 1\}, \\
\operatorname{Ker}(\gamma)=\operatorname{Im}(\bar{\gamma})=K \cdot c\{b, c\}^{*}\{a, 1\} \oplus K \cdot(a-1), \\
\operatorname{Ker}(\bar{\gamma})=\operatorname{Im}(\gamma)=K \cdot b\{b, c\}^{*}\{a, 1\} \oplus K \cdot(a+1) .
\end{gathered}
$$

In fact, let $x$ be an element written as (25) and suppose that $\theta(x)=x a+a x=0$. Then we have

$$
x a+a x=\Sigma\left(k_{i}+\ell_{i}\right) b x_{i}(a+1)+\sum\left(\ell_{i}^{\prime}-k_{i}^{\prime}\right) c x_{i}(a-1)+2 \ell a+2 k=0,
$$

and hence

$$
k=\ell=0, k_{i}+\ell_{i}=0, k_{i}^{\prime}=\ell_{i}^{\prime}
$$

for any $i$. It follows that $\operatorname{Ker}(\theta)$ is equal to the right-hand side of (29). On the other hand, for $x \in A$ given as (25) we have

$$
\bar{\theta}(z)=x a-a x=-\Sigma\left(k_{i}-\ell_{i}\right) b x_{i}(a-1)+\sum\left(k_{i}^{\prime}+\ell_{i}^{\prime}\right) c x_{i}(a+1) .
$$

This implies that $\operatorname{Im}(\bar{\theta})$ is also equal to the right-hand side of (29). The other equalities can be shown similarly.

From (27), (28) and equalities (29)-(32), we see that

$$
\operatorname{Ker}\left(\partial^{*}\right)=\operatorname{Im}\left(\bar{\partial}^{*}\right), \operatorname{Ker}\left(\bar{\partial}^{*}\right)=\operatorname{Im}\left(\partial^{*}\right),
$$

and we find

$$
H^{n}(A)=0
$$

for every $n \geq 2$.

Next we calculate $H^{1}(A)$. Since

$$
\partial_{1}(x)=\left(\bar{\theta}(x), \bar{\theta}_{2}(x)\right),
$$


where the $K$-linear mapping $\bar{\theta}_{2}: A \rightarrow A^{2}$ is defined by $\bar{\theta}_{2}(x)=(x b-b x, x c-c x)$ for $x \in A$, we have

$$
H^{1}(A)=\frac{\operatorname{Ker}(\partial)}{\operatorname{Im}\left(\partial_{1}\right)} \cong \frac{\operatorname{Ker}(\bar{\gamma}) \oplus \operatorname{Ker}(\gamma)}{\bar{\theta}_{2}(\operatorname{Ker}(\bar{\theta}))} .
$$

Let $x \in \operatorname{Ker}(\bar{\theta})$, then, by (30), $x$ is written as

$$
x=\sum k_{i} b x_{i}(a+1)+\sum \ell_{i} c x_{i}(a-1)+k a+\ell .
$$

We have

$$
x b-b x=\sum k_{i} b\left(2 x_{i} b-b x_{i}(a+1)\right)-\sum \ell_{i} b c x_{i}(a-1)-k b(a-1) .
$$

Thus, we can show that $b^{2}, b^{3}, \ldots, b^{i}, \ldots$ are linearly independent elements in $\operatorname{Ker}(\bar{\gamma})$ modulo $\{x b-b x \mid x \in \operatorname{Ker}(\bar{\theta})\}$. In virtue of (33), we find that $H^{1}(A)$ is infinite dimensional over $K$;

$$
H^{1}(A)=K^{\infty}
$$

Finally, it is easy to see that

$$
H^{0}(A)=\operatorname{Ker}\left(\partial_{1}\right)=K \text {. }
$$

Next, suppose $\operatorname{char}(K)=2$. In this case, by the generator change $a \rightarrow a+1$, we have a new presentation $K \Sigma^{*} / I\left(G^{\prime}\right)$ with $G^{\prime}=\left\{a^{2}, a b, a c\right\}$ for $A$. Using this Gröbner base $G^{\prime}$, we have a free resolution

$$
\cdots \stackrel{\partial}{\rightarrow} A \cdot \Sigma \cdot A \stackrel{\partial}{\rightarrow} \cdots \stackrel{\partial}{\rightarrow} A \cdot \Sigma \cdot A \stackrel{\partial_{1}}{\rightarrow} A[] A \stackrel{\epsilon}{\rightarrow} A,
$$

where the differentiation $\partial$ is given by

$$
\partial([a])=[a] a+a[a], \partial([b])=[a] b+a[b], \partial([c])=[a] c+a[c] .
$$

Using this resolution, though we omit the calculation, we can get

$$
H^{0}(A)=K
$$

and

for $n \geq 1$.

$$
H^{n}(A)=K^{\infty}
$$

Summarizing, we have

$$
\begin{gathered}
H^{0}(A)=K, H^{1}(A)=K^{\infty}, \\
H^{n}(A)= \begin{cases}0 & \text { if } \operatorname{char}(K) \neq 2, \\
K^{\infty} & \text { if } \operatorname{char}(K)=2,\end{cases}
\end{gathered}
$$

for $n \geq 2$.

Example 8.2. Let $U$ be an overlap-free subset of $\Sigma^{*} \backslash\{1\}$, that is,

(i) any $u \in U$ is not a subword of another word in $U$, and

(ii) any $u$ and $v$ in $U$ do not overlap, that is, $u=u^{\prime} t$ and $v=t v^{\prime}$ with $u^{\prime}, v^{\prime}, t \in \Sigma^{*}$ imply $t=1$.

Let $G$ be a monic subset of $F=K \Sigma^{*}$ such that the mapping sending $g$ to $\operatorname{lt}(g)$ is a bijection from $G$ to $U$. Then $G$ is a normalized Gröbner base on $F$. There is no critical pairs for $G$ by condition (ii). Thus, $C_{n}=\emptyset$ for all $n \geq 3$ in Theorem 7.6, and we have a resolution

$$
0 \rightarrow A \cdot U \cdot A \rightarrow A \cdot \Sigma \cdot A \rightarrow A \cdot[] \cdot A \rightarrow A .
$$

Hence, $H^{n}(A)=0$ for $n \geq 3$. 
Example 8.3. Let $U$ be a subset of $\Sigma^{\geq 2}$ satisfying (i) in Example 8.2. Then, $U$ is a normalized Gröbner base considered as a subset of $F$ consisting of only monic monomials. We have a monomial algebra $A=F / I(U)$ with augmentation mapping $\bar{\epsilon}: A \rightarrow K$ defined by $\bar{\epsilon}(a)=0$ for all $a \in \Sigma$. Here, we assume that $U$ satisfies the following additional condition:

(iii) There are no words $u, v, w, x \in U, z_{1}, z_{2}, z_{3}, z_{5}, z_{6}, z_{7} \in \Sigma^{+}$and $z_{4} \in \Sigma^{*}$ such that $u=z_{1} z_{2} z_{3}, v=z_{2} z_{3} z_{4} z_{5}, w=z_{3} z_{4} z_{5} z_{6}$ and $x=z_{5} z_{6} z_{7}$.

For $n \geq 1$ let $C_{n}$ be the set of sequences $\left[a, u_{1}, \ldots, u_{n-1}\right]$ of length $n$ such that $a u_{1} \in U$ and $u_{i} u_{i+1}$ is a minimal $U$-reducible word, that is, $u_{i}=u_{i}^{\prime} w_{i} \in U$ for some $w_{i} \in \Sigma^{*}$ and $w_{i} u_{i+1}$ is an only subword of $u_{i} u_{i+1}$ from $U$ for $i=1, \ldots, n-2$. Suppose that $n \geq 3$ and let $w_{1} u_{2}=b u_{2}^{\dagger}$ with $b \in \Sigma$ and $u_{2}^{\dagger} \in \Sigma^{+}$. By condition (iii) we see $\left[b, u_{2}^{\dagger}, u_{3}, \ldots, u_{n-1}\right] \in C_{n-1}$.

Now, our construction gives a resolution

$$
\cdots \rightarrow A \cdot C_{n} \cdot A \stackrel{\partial_{n}}{\rightarrow} A \cdot C_{n-1} \cdot A \rightarrow \cdots \rightarrow A \cdot C_{1} \cdot A \stackrel{\partial_{1}}{\rightarrow} A \cdot[] \cdot A \rightarrow A
$$

with

$$
\begin{gathered}
\partial_{1}([a])=[] a-a[], \\
\partial_{2}\left(\left[a, u_{1}\right]\right)=[a] u_{1}+a\left[a_{2}\right] a_{3} \cdots a_{l}+\cdots+a a_{2} \cdots a_{l-1}\left[a_{l}\right]
\end{gathered}
$$

where $u_{1}=a_{2} \cdots a_{l}$, and for $n \geq 3$,

$$
\partial_{n}\left(\left[a, u_{1}, \cdots, u_{n-1}\right]\right)=\left[a, u_{1}, \cdots, u_{n-2}\right] u_{n-1}+(-1)^{n} a u_{1}^{\prime}\left[b, u_{2}^{\dagger}, \cdots, u_{n-1}\right] .
$$

Tensoring the complex (34) with $K$ over $A$ from both sides, we have a complex

$$
\cdots \rightarrow K \cdot C_{n} \stackrel{\bar{\partial}_{n}}{\rightarrow} K \cdot C_{n-1} \rightarrow \cdots \rightarrow K \cdot C_{1} \stackrel{\bar{\partial}_{1}}{\rightarrow} K \cdot[] \rightarrow K,
$$

where all the differentiations $\bar{\partial}_{n}$ are 0 . Hence,

$$
\operatorname{Tor}_{n}^{A}(K, K)=K^{c_{n}}
$$

for all $n \geq 1$, where $c_{n}$ is the cardinality of the set $C_{n}$. This, in particular, means that the resolution (34) is minimal.

Example 8.4. Let $\Lambda=K \cdot \Sigma$ be a Lie algebra over $K$, which is a free $K$-module generated by a well-ordered set $(\Sigma,>)$. For different $a, b \in \Sigma$, the Lie product $[a, b]$ is a linear combination $\ell(a, b)$ of elements of $\Sigma$ over $K$. The enveloping algebra $A$ of $L$ is the associative algebra which is the quotient algebra of $F=K \cdot \Sigma^{*}$ modulo the ideal generated by $G=\{a b-b a-\ell(a, b) \mid a>b\}$. We can easily check that $G$ is a normalized Gröbner base for $A$ with respect to the order $\succ_{\text {llex }}$ defined through the order $>$ on $\Sigma$. For $n \geq 0$ let $C_{n}$ be the set of words $a_{1} \cdots a_{n} \in \Sigma^{*}$ with $a_{1}<a_{2} \cdots<a_{n}$ of length $n$. Then, we have an $A$-bimodule resolution

$\cdots \rightarrow A \cdot C_{n} \cdot A \stackrel{\partial_{n}}{\rightarrow} A \cdot C_{n-1} \cdot A \rightarrow \cdots \rightarrow A \cdot C_{2} \cdot A \stackrel{\partial_{2}}{\rightarrow} A \cdot \Sigma \cdot S \stackrel{\partial_{1}}{\rightarrow} A[] A \rightarrow A \rightarrow 0$.

Here, $\operatorname{Ker}\left(\partial_{n-1}\right)$ has a Gröbner base $H_{n}=\left\{h_{v} \mid v \in C_{n}\right\}$ on $F \cdot C_{n-1} \cdot F$ modulo $G$ such that $\operatorname{lt}\left(h_{v}\right)=\left[a_{1} a_{2} \cdots a_{n-1}\right] a_{n}$, and $\partial_{p}([v])=\rho_{C_{n-1}}\left(h_{v}\right)$.

To describe the differential mapping $\partial$ neatly we identify the $K$-space $K \cdot C_{n}$ with the component $\bigwedge_{n}(\Lambda)$ of degree $n$ of the exterior algebra $\Lambda(\Lambda)$ of $\Lambda$ over $K$. Following our construction we get the differential mapping $\partial: A \cdot \Lambda(\Lambda) \cdot A=$ 
$A \otimes_{K} \bigwedge(\Lambda) \otimes_{K} A \rightarrow A \cdot \Lambda(\Lambda) \cdot A$ given by

$$
\begin{aligned}
\partial\left(x_{1} \wedge \cdots \wedge x_{n}\right)= & \sum(-1)^{i} x_{i} \otimes x_{1} \wedge \cdots \wedge \check{x}_{i} \wedge \cdots x_{n} \\
& -\sum(-1)^{i} x_{1} \wedge \cdots \wedge \check{x}_{i} \wedge \cdots \wedge x_{n} \otimes x_{i} \\
& +\sum_{i<j}(-1)^{i+j} \ell\left(x_{i}, x_{j}\right) \wedge x_{1} \wedge \cdots \wedge \check{x}_{i} \wedge \cdots \wedge \check{x}_{j} \wedge \cdots \wedge x_{n} .
\end{aligned}
$$

This is a two-sided version of the standard complex for $\Lambda$ (7], Chap. 8, Theorem 7.1).

For example, let $A$ be the enveloping algebra of the Lie algebra $s o(3)$ generated by $\Sigma=\{a, b, c\}$ subject to the relations

$$
[a, b]=c,[b, c]=a,[a, c]=-b .
$$

Then, we have the Gröbner base

$$
G=\left\{g_{1}=a b-b a-c, g_{2}=b c-c b-a, g_{3}=a c-c a+b\right\}
$$

on $F=K \Sigma^{*}$ for $A$ and the resolution

$$
0 \rightarrow A \cdot[h] \cdot A \stackrel{\partial_{3}}{\rightarrow} A \cdot G \cdot A \stackrel{\partial_{2}}{\rightarrow} A \cdot \Sigma \cdot A \stackrel{\partial_{1}}{\rightarrow} A[] A \rightarrow A,
$$

where

$$
\begin{gathered}
\partial_{2}\left(\left[g_{1}\right]\right)=[a] b+a[b]-[b] a-b[a]-[c], \\
\partial_{2}\left(\left[g_{2}\right]\right)=[b] c+b[c]-[c] b-c[b]-[a], \\
\partial_{2}\left(\left[g_{3}\right]\right)=[a] c+a[c]-[c] a-c[a]+[b], \\
\partial_{3}([h])=\left[g_{1}\right] c-c\left[g_{1}\right]-a\left[g_{2}\right]+\left[g_{2}\right] a+b\left[g_{3}\right]-\left[g_{3}\right] b .
\end{gathered}
$$

Finally, we consider an algebra that admits no finite Gröbner base. The following example is taken from Kobayashi [17].

Example 8.5. Let $\Sigma=\{a, b, c\}$. Let $I$ be the ideal of $F=K \cdot \Sigma^{*}$ generated by $\{b a-a b, b c-a c a, a c c\}$ and let $A=F / I$. Consider the weight function $\omega: \Sigma^{*} \rightarrow \mathbf{N}$ defined by $\omega(a)=\omega(c)=1, \omega(b)=2$. Let $\preceq$ be the order on $\Sigma^{*}$ given as follows. For $x, y \in \Sigma^{*}$ define $x \preceq y$ if and only if either $\omega(x)<\omega(y)$ or $\omega(x)=\omega(y)$ and $x$ is lexicographically smaller than $y$. By the Knuth-Bendix completion procedure [15] (see also Kapur and Narendran [14]) based on this well-ordering, we have a normalized infinite Gröbner base of $I$ :

$$
\left\{b a-a b, b c-a c a, a^{n} c a^{n-1} c \mid n=1,2, \ldots\right\} .
$$

In 17] it is proved that $A$ has no Gröbner base $G$ such that $\operatorname{Dom}(G)$ forms a regular language, a fortiori, $A$ has no finite Gröbner base. Set

$$
G=\left\{\alpha, \beta, \gamma_{n} \mid n=1,2, \ldots\right\},
$$

where $\alpha, \beta$ and $\gamma_{n}$ correspond to the elements $b a-a b, b c-a c a$ and $a^{n} c a^{n-1} c$, respectively, and for $p \geq 1$ set

$$
\begin{aligned}
H_{p}=\left\{\alpha\left(m_{1}, \ldots, m_{p}\right), \gamma\left(n_{1}, \ldots, n_{p}, n_{p+1}\right) \mid m_{1}>\cdots>m_{p}\right. & >0, \\
n_{1} & \left.>\cdots>n_{p+1}>0\right\} .
\end{aligned}
$$

Our construction gives the following resolution of $A$ :

$$
\cdots \rightarrow A \cdot H_{p} \cdot A \stackrel{\partial_{p+2}}{\rightarrow} A \cdot H_{p-1} \cdot A \rightarrow \cdots \stackrel{\partial_{4}}{\rightarrow} A \cdot H_{1} \cdot A \stackrel{\partial_{3}}{\rightarrow} A \cdot G \cdot A \stackrel{\partial_{2}}{\rightarrow} A \cdot \Sigma \cdot A \stackrel{\partial_{1}}{\rightarrow} A \cdot[] \cdot A \stackrel{\epsilon}{\rightarrow} A,
$$


where

$$
\begin{gathered}
\partial_{1}([a])=[] a-a[], \partial_{1}([b])=[] b-b[], \partial_{1}([c])=[] c-c[], \\
\partial_{2}([\alpha])=[b] a+b[a]-[a] b-a[b], \partial_{2}([\beta])=[b] c+b[c]-[a] c a-a[c] a-a c[a], \\
\partial_{2}\left(\left[\gamma_{n}\right]\right)=[a] a^{n-1} c a^{n-1} c+\cdots+a^{n-1}[a] c a^{n-1} c+a^{n}[c] a^{n-1} c+a^{n} c[a] a^{n-2} c \\
+\cdots+a^{n} c a^{n-2}[a] c+a^{n} c a^{n-1}[c], \\
\partial_{3}([\alpha(n)])=[\alpha] a^{n-1} c a^{n-1} c-b\left[\gamma_{n}\right]+\left[\gamma_{n+1}\right]+a[\alpha] a^{n-2} c a^{n-1} c \\
+\cdots+a^{n-1}[\alpha] c a^{n-1} c+a^{n}[\beta] a^{n-1} c \\
\partial_{3}([\gamma(n, m)])=\left[\gamma_{n}\right] a^{m-1} c-a^{n} c a^{n-m-1}\left[\gamma_{m}\right],
\end{gathered}
$$

and for $p \geq 2$,

$$
\begin{aligned}
& \partial_{p+2}\left(\left[\alpha\left(n_{1}, n_{2}, \ldots, n_{p}\right)\right]\right)=\left[\alpha\left(n_{1}, \ldots, n_{p-1}\right)\right] a^{n_{p}} c \\
& \quad+(-1)^{p} b\left[\gamma\left(n_{1}, n_{2}, \ldots, n_{p}\right)\right]+(-1)^{p-1}\left[\gamma\left(n_{1}+1, n_{2}, \ldots, n_{p}\right)\right], \\
& \partial_{p+2}\left(\left[\gamma\left(n_{1}, n_{2}, \ldots, n_{p}, n_{p+1}\right)\right]\right)=\left[\gamma\left(n_{1}, n_{2}, \ldots, n_{p}\right)\right] a^{n_{p+1}} c \\
& \quad+(-1)^{p} a^{n_{1}} c a^{n_{1}-n_{2}-1}\left[\gamma\left(n_{2}, \ldots, n_{p}, n_{p+1}\right)\right] .
\end{aligned}
$$

Via the augmentation mapping $\bar{\epsilon}: A \rightarrow K$ defined by $\bar{\epsilon}(a)=\bar{\epsilon}(b)=\bar{\epsilon}(c)=0$, $K$ is the $A$-bimodule. Tensoring (35) with $K$ over $A$ from both sides, we have a complex

$$
\cdots \rightarrow K \cdot H_{p} \stackrel{\bar{\partial}_{p}}{\rightarrow} K \cdot H_{p-1} \rightarrow \cdots \rightarrow K \cdot H_{1} \stackrel{\bar{\partial}_{3}}{\rightarrow} K \cdot G \stackrel{\bar{\partial}_{2}}{\rightarrow} K \cdot \Sigma \stackrel{\bar{\partial}_{1}}{\rightarrow} K \cdot[] \rightarrow 0,
$$

where $\bar{\partial}_{1}=\bar{\partial}_{2}=0, \bar{\partial}_{3}([\alpha(n)])=\left[\gamma_{n+1}\right], \partial_{3}([\gamma(n, m)])=0$, and

$$
\begin{gathered}
\bar{\partial}_{p+2}\left(\left[\alpha\left(n_{1}, n_{2}, \ldots, n_{p}\right)\right]\right)=(-1)^{p-1}\left[\gamma\left(n_{1}+1, n_{2}, \ldots, n_{p}\right)\right], \\
\bar{\partial}_{p+2}\left(\left[\gamma\left(n_{1}, n_{2}, \ldots, n_{p}, n_{p+1}\right)\right]\right)=0
\end{gathered}
$$

for $p \geq 2$. Thus, we have

$$
\operatorname{Tor}_{n}^{A}(K, K)=\operatorname{Ker}\left(\bar{\partial}_{n}\right) / \operatorname{Im}\left(\bar{\partial}_{n+1}\right)= \begin{cases}K & \text { if } n=0, \\ K^{3} & \text { if } n=1,2, \\ K^{\infty} & \text { if } n \geq 3\end{cases}
$$

Remark 8.6. The Hochschild cohomology of finite dimensional algebras over a field has been extensively studied (see [10, [19] for example). Since a finite dimensional algebra has a finite Gröbner base, we can apply our method, but it is not a clever way. For example, even the one-arrow quiver algebra is presented as the quotient of the free algebra generated by two elements $a$ and $b$ modulo the ideal generated by the Gröbner base $\left\{a^{2}, a b, b^{2}-b, b a-a\right\}$. The resolution constructed on this base is not neat at all. Instead, by considering Gröbner bases on path algebras and projective bimodules, we can construct simpler resolutions for algebras presented as quotients of path algebras. This will be discussed in a forthcoming paper.

\section{REFERENCES}

[1] D.J. Anick, On the homology of associative algebras, Trans. Amer. Math. Soc. 296 (1987), 641-659. MR87i:16046

[2] T. Becker and V. Weispfenning, Gröbner Bases, Springer, 1993. MR95e:13018

[3] G. Bergman, The diamond lemma for ring theory, Adv. Math. 29 (1978), 178-218. MR $81 \mathrm{~b}: 16001$

[4] R.V. Book and F. Otto, String-Rewriting Systems, Springer, 1993. MR94f:68108 
[5] B. Buchberger, Ein Algorithmus zum Auffinden der Basiselemente des Restklassennringes nach einem nulldimensionalen Polynomideal, Ph.D. Thesis, Innsbruck, 1965.

[6] B. Buchberger, Gröbner bases: an algorithmic method in polynomial ideal theory, in: Recent Trends in Multidimensional System Theory, Reidel Publishing (1985), 184-232.

[7] H. Cartan and S Eilenberg, Homological Algebra, Princeton Univ. Press, 1956. MR 17:1040e

[8] D. Cox, J. Little and D. O'Shea, Ideals, Varieties, and Algorithms, Springer, 1991. MR $93 \mathrm{j}: 13031$

[9] E. Green, T. Mora and V. Ufnarovski, The non-commutative Gröbner freaks, in: Symbolic Rewriting Techniques, Birkhäuser (1998), 93-104. MF99i:16050

[10] D. Happel, Hochschild cohomology of finite-dimensional algebras, Lect. Notes Math. 1404, Springer (1989), 108-126. MR.91b:16012

[11] G. Hochschild, On the cohomology groups of an associative algebra, Ann. Math. 46 (1945), 58-67. MF 6:114f

[12] G. Huet, Confluent reductions: abstract properties and applications to term rewriting systems, J. ACM 27 (1980), 797-821. MR82a:68090

[13] A. Kandre-Rody and V. Weispfenning, Noncommutative Gröbner bases in algebra of solvable type, J. Symb. Comp. 9 (1990), 1-26. MF 91e:13025

[14] D. Kapur and P. Narendran, The Knuth-Bendix completion procedure and Thue systems, SIAM J. Comp. 14 (1985), 1052-1072. MR87f:03103

[15] D.E. Knuth and P.B. Bendix, Simple word problems in universal algebras, in: Computational Problems in Abstract Algebra, Pergamon (1970), 263-297. MR41:134

[16] Y. Kobayashi, Complete rewriting systems and homology of monoid algebras, J. Pure Appl. Algebra 65 (1990), 264-275. MR92d:18003

[17] Y. Kobayashi, A finitely presented monoid which has solvable word problem but has no regular complete presentation, Theoret. Comp. Sci. 146 (1995), 321-329. MR96d:20058

[18] H. Li, Noncommutative Gröbner Bases and Filtered-Graded Transfer, Lect. Notes Math. 1795, Springer, 2002. MR2003i:16065

[19] A. C. Locateli, Hochschild cohomology of truncated quiver algebras, Comm. Algebra 27 (1999), 645-664. MR2000d:16014

[20] K. Madlener and B. Reinert, Relating rewriting techniques on monoids and rings: congruences on monoids and ideals in monoid rings, Theoret. Comp. Sci. 208 (1998), 3-31. MR $99 \mathrm{~m}: 68102$

[21] F. More, Gröbner bases for noncommutative polynomial rings, In: AAECC3, Lect. Notes Comp. Sci. 229, 253-262, Springer, 1986.

Department of Information Science, Toho University, Funabashi 274-8510, Japan

E-mail address: kobayasi@is.sci.toho-u.ac.jp 